\title{
Logics of Formal Inconsistency enriched with replacement: an algebraic and modal account*
}

\author{
Walter Carnielli ${ }^{1}$, Marcelo E. Coniglio ${ }^{1,2}$ and David Fuenmayor ${ }^{3}$ \\ ${ }^{1}$ Centre for Logic, Epistemology and the History of Science - CLE \\ University of Campinas, Brazil \\ ${ }^{2}$ Institute of Philosophy and the Humanities - IFCH \\ University of Campinas, Brazil \\ ${ }^{3}$ Department of Computer Science \\ University of Luxembourg, Luxembourg \\ Email: \{walterac, coniglio\}@unicamp.br and david.fuenmayor@uni.lu
}

\begin{abstract}
It is customary to expect from a logical system that it can be algebraizable, in the sense that an algebraic companion of the deductive machinery can always be found. Since the inception of da Costa's paraconsistent calculi, algebraic equivalents for such systems have been sought. It is known, however, that these systems are not self-extensional (i.e., they do not satisfy the replacement property). More than this, they are not algebraizable in the sense of Blok-Pigozzi. The same negative results hold for several systems of the hierarchy of paraconsistent logics known as Logics of Formal Inconsistency (LFIs). Because of this, several systems belonging to this class of logics are only characterizable by semantics of a non-deterministic nature. This paper offers a solution for two open problems in the domain of paraconsistency, in particular connected to algebraization of LFIs, by extending with rules several LFIs weaker than $C_{1}$, thus obtaining the replacement property (that is, such LFIs turn out to be self-extensional). Moreover, these logics become algebraizable in the standard Lindenbaum-Tarski's sense by a suitable variety of Boolean algebras extended with additional operations. The weakest LFI satisfying replacement presented here is called $\mathbf{R m b C}$, which is obtained from the basic LFI called $\mathbf{m b C}$. Some axiomatic extensions of $\mathbf{R} \mathbf{m b C}$ are also studied. In addition, a neighborhood semantics is defined for such systems. It is shown that $\mathbf{R m b C}$ can be defined within the minimal bimodal non-normal logic $\mathbf{E} \oplus \mathbf{E}$ defined by the fusion of the non-normal modal logic $\mathbf{E}$ with itself. Finally, the framework is extended to first-order languages. RQmbC, the quantified extension of $\mathbf{R m b C}$, is shown to be sound and complete w.r.t. the proposed algebraic semantics.
\end{abstract}

\footnotetext{
${ }^{*}$ This article has been accepted for publication and will appear in a revised form in The Review of Symbolic Logic, published by Cambridge University Press on behalf of the Association for Symbolic Logic.

(C)Association for Symbolic Logic, 2021
} 


\section{Contents}

1 Introduction: The quest for the algebraic counterpart of paraconsistency 2

2 The logic RmbC 4

3 Adding replacement to extensions of mbC: A solution to an open problem 10

4 Limits for replacement while preserving paraconsistency

5 Neighborhood semantics for RmbC

$5.1 \mathrm{RmbC}$ is definable within the minimal bimodal modal logic . . . . . . . . . . 17

5.2 Neighborhood models for axiomatic extensions of $\mathbf{R m b C} \ldots \ldots \ldots$

6 Truth-preserving (or global) semantics 22

7 Extension to first-order logics 24

7.1 BALFI semantics for RQmbC . . . . . . . . . . . . . . 25

7.2 Completeness of RQmbC w.r.t. BALFI semantics . . . . . . . . . . 27

8 Conclusion, and significance of the results 30

References 31

\section{Introduction: The quest for the algebraic counterpart of para- consistency}

It is customary to expect from a logical system that it can be algebraizable, in the sense that an algebraic companion of the deductive machinery can always be found. When this happens, several logical problems can be faithfully and conservatively translated into some given algebra, and then algebraic tools can be used to tackle them. This happens so naturally with the brotherhood between classical logic and Boolean algebra, that a similar relationship is expected to hold for non-standard logics as well. And indeed, this holds for some, but not for all logics. In any case, the task of finding such an algebraic counterpart is far from trivial. The intuitive idea behind the search for algebraization for a given logic system, generalizing the pioneering proposal of Lindenbaum and Tarski, usually starts by trying to find a congruence on the set of formulas that could be used to produce a quotient algebra, defined over the algebra of formulas of the logic.

One of the favorite methods to set up congruences is to check the validity of a fundamental property called replacement or (IpE) (acronym for intersubstitutivity by provable equivalents. Intuitively, (IpE) states that if $\alpha$ and $\beta$ are logically equivalent, then the replacement in a formula $\gamma$ of any ocurrences of $\alpha$ by $\beta$ produces a formula logically equivalent to $\gamma$. A logic enjoying replacement is usually called self-extensional.

Finding an algebraization for the logics of the hierarchy $C_{n}$ of paraconsistent logics, introduced in da Costa (1963), constitutes a paradigmatically difficult case. Recall that a logic with a negation $\neg$ is paraconsistent if it can incorporate contradictions (w.r.t. $\neg$ ) that do not trivialize the system (different from what happens, for instance, with the classical or intuitionistic negation). The idea of da Costa's systems is to define, for each $C_{n}$, an unary connective $\circ_{n}$ (where ${ }^{\circ} \alpha$ means that $\alpha$ is 'well-behaved', or 'classically behaved' in $C_{n}$ ) in the following sense: in 
spite of there existing formulas $\alpha$ and $\beta$ such that $\beta$ does not follow from the contradictory set $\{\alpha, \neg \alpha\}, \beta$ is always derivable in $C_{n}$ from $\left\{\alpha, \neg \alpha, \circ_{n} \alpha\right\}$, for every $\alpha$ and $\beta$ (thus, the explosion principle of negation is only guaranteed for contradictions involving well-behaved formulas). For instance, well-behavedness is defined by $\circ_{1} \alpha \stackrel{\text { def }}{=} \neg(\alpha \wedge \neg \alpha)$ in $C_{1}$, the first logic of da Costa's family. The approach to paraconsistency of da Costa was afterwards generalized in Carnielli and Marcos (2002) through the notion of Logics of Formal Inconsistency (LFIs), in which the operator of well-behavedness (rebaptized as consistency operator, and denoted by $\circ$ ) is allowed to be a primitive one.

It is known since a long time that (IpE) does not hold for $C_{1}$ (see da Costa and Guillaume (1964. Corollary to Theorem 1); a proof for the calculi $C_{n}$ can be found in Urbas (1989, Theorem 1)). As a consequence of this failure, a direct algebraization (in the sense of LindenbaumTarski) for this logic is not possible. This blocks the way for other, weaker calculi of the hierarchy $C_{n}$, since when one logic is algebraizable, so are its extensions. But there are further possibilities for algebraization, and the search continued until a proof was presented in Mortensen (1980), establishing that no non-trivial quotient algebra is definable for $C_{1}$, or for any logic weaker than $C_{1}$. This entails that $C_{1}$ (together with its subsystems) is not algebraizable in the general sense of Blok and Pigozzi (1989) (a simpler proof of this fact was obtained in Lewin et al. (1991)). This result was generalized in Carnielli and Marcos (2002, Theorem 3.83) to Cila, the presentation of $C_{1}$ in the language of the LFIs (where the consistency conective $\circ$ is primitive instead of being defined in terms of the other connectives). We obtain as a consequence that no such algebraization is possible for any other of the LFIs weaker than Cila to be discussed in Section 3 (i.e., the systems $\mathbf{m b C}$, mbCciw, bC and $\mathbf{C i}$ studied in Carnielli and Marcos (2002), Carnielli et al. (2007), and Carnielli and Coniglio (2016a)). The same reasoning applies to every calculus $C_{n}$ in the infinite da Costa's hierarchy, since they are weaker than $C_{1}$.

Some extensions of $C_{1}$ having non-trivial quotient algebras have been proposed in the literature. In Mortensen (1989), for instance, it is proposed an infinite number of intermediate logics between $C_{1}$ and classical logic called $C_{n /(n+1)}$, for $n \geq 1$. In each $C_{n /(n+1)}$ it is possible to define a non-trivial logical congruence $\sim_{n}$ by means of a formula $\delta_{n}(\alpha, \beta)$ such that $\alpha \sim_{n} \beta$ iff $\vdash_{C_{n /(n+1)}} \delta_{n}(\alpha, \beta)$ (see Mortensen, 1989. Theorem 4.4). However, this feature is strictly weaker than being self-extensional.

Some other types of algebraic-like counterparts have been investigated, for instance, in Carnielli and de Alcantara (1984) and Seoane and de Alcantara (1991) a class of structures for the logic $C_{1}$ (called da Costa algebras) was defined, permitting a Stone-like representation theorem. In this way, every da Costa algebra is isomorphic to a paraconsistent algebra of sets, making $C_{1}$ closer to traditional mathematical objects. On the other hand, in Coniglio and Toledo (2020) a semantical characterization (which constitutes a decision procedure) is given for the $\operatorname{logics} C_{n}$ in terms of restricted non-deterministic matrices. These structures are nondeterministic matrices (that is, logical matrices in which the connectives can take several values instead of a single one; $\mathrm{cf}$. Avron (2005)) such that the valuations must satisfy some (decidable) conditions.

To the best of our knowledge, the search for da Costa-like paraconsistent systems satisfying (IpE) started in the studies by Urbas (1987) and Sylvan (1990). The former, a PhD thesis supervised by Sylvan, analyzes (IpE) for some subsystems of the hierarchy of $J$-systems introduced in Arruda and da Costa (1970). The latter proposes an extension of da Costa's $C_{\omega}$ by adding the inference rule of contraposition, which guarantees replacement. In the realm of LFIs, the situation is more complicated: it can be proved that, for some subclasses of LFIs, (IpE) is unattainable (while preserving paraconsistency), as shown in Carnielli and Marcos (2002, Theorem 3.51) with respect to some axiomatic extensions of the logic $\mathbf{C i}$, one of the central systems of the family of LFIs which is much weaker than Cila.

Some interesting results concerning three-valued self-extensional paraconsistent logics were obtained in the literature, in connection with the limitative result mentioned above (namely, 
Carnielli and Marcos, 2002, Theorem 3.51). In Avron and Béziau (2017) it was shown that no three-valued paraconsistent logic having an implication can be self-extensional. On the other hand, in Avron (2017) it was shown that there is exactly one self-extensional three-valued paraconsistent logic defined in a signature having conjunction, disjunction and negation. For paraconsistent logics in general, it was shown in Béziau (1998) that no paraconsistent negation $\neg$ satisfying the law of double negation and such that the schema $\neg(\varphi \wedge \neg \varphi)$ is valid can satisfy (IpE).

Nevertheless, a problem has remained open: to obtain (IpE) for extensions of $\mathbf{C i}$ by the addition of weaker forms of contraposition deduction rules while preserving their paraconsistent character, as discussed in Carnielli and Marcos (2002, Subsection 3.7). The challenge was to find extensions of $\mathbf{b C}$ and $\mathbf{C i}$ which would satisfy (IpE) and still keep their paraconsistent character. This paper meets this challenge in a minimal way: we define the logic $\mathbf{R m b C}$, an extension by rules of $\mathbf{m b C}$, and two suitable extensions of $\mathbf{R m b C}$, the logics $\mathbf{R b C}$ and $\mathbf{R C i}$ (respectively, extensions of $\mathbf{b C}$ and $\mathbf{C i}$ ) that solve the open problem. Details and exemplary model structures are presented in Examples 3.9 and 3.10 in Section $3{ }^{1}$

The (minimal) logic $\mathbf{R m b C}$ and its axiomatic extensions thus form a parallel family of LFIs featuring self-extensionality. We introduce for them, in Section 2, a new kind of semantic structures: the Boolean algebras with LFI operators, or BALFIs. Moreover, RmbC is proved to be sound and complete w.r.t. the class of BALFIs. In Section 3 we consider axiomatic extensions of $\mathbf{R m b C}$ and give solutions to the open problems mentioned above. Section 4 studies some limits for self-extensionality for extensions of $\mathbf{R m b C}$ under the condition of paraconsistency.

The paper also explores some other directions. Section 5 proposes a neighborhood semantics for $\mathbf{R m b C}$ as a special class of BALFIs defined on powerset Boolean algebras. Again, $\mathbf{R m b C}$ is proved to be sound and complete w.r.t. these neighborhood models. Moreover, in Section 5.1 it is proved that $\mathbf{R m b C}$ can be defined within the minimal bimodal non-normal modal logic E. Such a neighborhood semantics is also proposed for axiomatic extensions of $\mathbf{R m b C}$ in Section 5.2 .

The notion of logical consequence used in BALFI semantics for RmbC, as well as in its neighborhood semantics (as defined in Section 5), is local instead of global (or degree-preserving instead of truth-preserving in the sense of Bou et al. (2009)). This requires adapting the usual definition of derivation from premises in a Hilbert calculus. In fact, we can, alternatively, consider a global (or truth-preserving) semantics, as it is usually done with algebraic semantics. This will leads us, in Section 6, to the logic $\mathbf{R} \mathbf{m b C} \mathbf{C}^{*}$, which is defined by the same Hilbert calculus as for $\mathbf{R} \mathbf{m b C}$, but where derivations from premises are defined as in the usual Hilbert calculi.

Section 7 is dedicated to extending $\mathbf{R m b C}$ to first-order languages, thus defining the logic RQmbC, which is proved, in Section 7.1 and Section 7.2, to be complete w.r.t. BALFI semantics.

\section{The logic RmbC}

The class of paraconsistent logics known as Logics of Formal Inconsistency (LFIs, for short) was introduced by Carnielli and Marcos (2002). In their simplest form, they feature a 'non-explosive' negation $\neg$, as well as a (primitive or derived) consistency connective $\circ$ which allows us to recover the principle of explosion (ex contradictione sequitur quodlibet) in a controlled way.

\footnotetext{
${ }^{1}$ We acknowledge the help of model finder Nitpick (Blanchette and Nipkow, 2010), integrated in the proof assistant Isabelle (Nipkow et al., 2002), in the task of generating suitable models for theories. By finding countermodels, Nitpick also spared us the Sisyphean task of trying to prove non-theorems. In order to encode $\mathbf{R m b C}$ and its extensions in the higher-order logic of Isabelle/HOL we employed the shallow semantical embedding approach by Benzmüller (2019). Formalization sources for selected parts of this work are available at: https://github.com/ davfuenmayor/LFIs. See also Fuenmayor (2020) for a recent Isabelle/HOL encoding of a generalized topological semantics for paraconsistent and paracomplete logics, which, in a sense, generalizes the neighbourhood semantics presented in Section 5
} 
Definition 2.1 Let $\mathbf{L}=\langle\Theta, \vdash\rangle$ be a Tarskian, finitary and structural logic defined over a propositional signature $\Theta$, which contains a negation $\neg$, and let $\circ$ be a (primitive or derived) unary connective. Then, $\mathbf{L}$ is said to be a Logic of Formal Inconsistency (LFI) with respect to $\neg$ and $\circ$ if the following holds:

(i) $\varphi, \neg \varphi \nvdash \psi$ for some $\varphi$ and $\psi$;

(ii) $\circ \varphi, \varphi, \neg \varphi \vdash \psi$ for every $\varphi$ and $\psi$;

(iii) there are two formulas $\alpha$ and $\beta$ such that

$$
\begin{aligned}
& \text { (iii.a) } \circ \alpha, \alpha \nvdash \beta ; \\
& \text { (iii.b) } \circ \alpha, \neg \alpha \nvdash \beta .
\end{aligned}
$$

Condition (i) above signals the non-validity of the classical principle of explosion. Condition (ii), also called principle of gentle explosion, characterizes LFIs in particular. Condition (iii) is required in order to satisfy condition (ii) in a non-trivial way. The hierarchy of LFIs studied in Carnielli et al. (2007) and Carnielli and Coniglio (2016a) starts from a logic called mbC, which extends positive classical logic $\mathbf{C P L}^{+}$by adding a negation $\neg$ and a primitive consistency operator $\circ$ satisfying minimal requirements in order to define an LFI.

DEFINITION 2.2 In what follows, the following signatures will be considered:

$$
\begin{aligned}
& \Sigma_{+}=\{\wedge, \vee, \rightarrow\} ; \\
& \Sigma_{B A}=\{\wedge, \vee, \rightarrow, \overline{0}, \overline{1}\} ; \\
& \Sigma=\{\wedge, \vee, \rightarrow, \neg, \circ\} ; \\
& \Sigma_{C}=\{\wedge, \vee, \rightarrow, \neg\} ; \\
& \Sigma_{C_{0}}=\{\wedge, \vee, \rightarrow, \neg, \overline{0}\} ; \\
& \Sigma_{C_{e}}=\{\wedge, \vee, \rightarrow, \neg, \overline{0}, \overline{1}\} ; \\
& \Sigma_{e}=\{\wedge, \vee, \rightarrow, \neg, \circ, \overline{0}, \overline{1}\} ; \\
& \Sigma_{m}=\{\wedge, \vee, \rightarrow, \sim, \square, \diamond\} ; \text { and } \\
& \Sigma_{b m}=\left\{\wedge, \vee, \rightarrow, \sim, \square_{1}, \diamond_{1}, \square_{2}, \diamond_{2}\right\} .
\end{aligned}
$$

If $\Theta$ is a propositional signature, then $\operatorname{For}(\Theta)$ will denote the (absolutely free) algebra of formulas over $\Theta$ generated by a given denumerable set $\mathcal{V}=\left\{p_{n}: n \in \mathbb{N}\right\}$ of propositional variables. Throughout this paper, logic systems will be presented by means of Hilbert calculi (in some cases with two possible definitions of derivations from premises: local and global), and a semantics will be given for them by means of algebraic structures, proving the soundness and completeness of each logic w.r.t. its semantics. For simplicity, any logic will be identified with its Hilbert calculus presentation (w.r.t. a given notion of derivation in that calculus).

Definition 2.3 (Classical Positive Logic) The classical positive logic $\mathbf{C P L}{ }^{+}$is defined over the language For $\left(\Sigma_{+}\right)$by the following Hilbert calculus ${ }^{2}$

\footnotetext{
${ }^{2}$ It is hard to pinpoint in the history of logic who first proposed an axiomatization for the full positive fragment of classical propositional calculus. H. B. Curry in a book written in 1959-1961 for a course (published as Curry (1963)) deals in his chapter 5 with the positive fragment of the propositional calculus (i.e., with conjunction, disjunction and implication, but without negation). Before that, Łukasiewicz (1950) and Henkin (1949), among other authors, studied proper fragments of positive logic.
} 


\section{Axiom schemas:}

$$
\begin{aligned}
& \alpha \rightarrow(\beta \rightarrow \alpha) \\
&(\alpha \rightarrow(\beta \rightarrow \gamma)) \rightarrow((\alpha \rightarrow \beta) \rightarrow(\alpha \rightarrow \gamma)) \\
& \alpha \rightarrow(\beta \rightarrow(\alpha \wedge \beta)) \\
&(\alpha \wedge \beta) \rightarrow \alpha \\
&(\alpha \wedge \beta) \rightarrow \beta \\
& \alpha \rightarrow(\alpha \vee \beta) \\
& \beta \rightarrow(\alpha \vee \beta) \\
&(\alpha \rightarrow \gamma) \rightarrow((\beta \rightarrow \gamma) \rightarrow((\alpha \vee \beta) \rightarrow \gamma)) \\
&(\alpha \rightarrow \beta) \vee \alpha
\end{aligned}
$$

Inference rule:

$$
\frac{\alpha \quad \alpha \rightarrow \beta}{\beta}
$$

DeFinition 2.4 The logic $\mathbf{m b C}$, defined over signature $\Sigma$, is obtained from $\mathbf{C P L}^{+}$by adding the following axiom schemas:

$$
\begin{gathered}
\alpha \vee \neg \alpha \\
\circ \alpha \rightarrow(\alpha \rightarrow(\neg \alpha \rightarrow \beta))
\end{gathered}
$$

The logic $\mathbf{m b C}$ is an LFI. Indeed, it is the minimal LFI extending $\mathbf{C P L} \mathbf{L}^{+}$.

Take into consideration the replacement property, namely: If $\alpha \leftrightarrow \beta$ is a theorem then $\gamma[p / \alpha] \leftrightarrow \gamma[p / \beta]$ is a theorem, for every formula $\gamma(p) 3^{3}$ It is well known that $\mathbf{m b C}$ does not satisfy the replacement property in general. However, it is easy to see that replacement holds in $\mathbf{m b C}$ for every formula $\gamma(p)$ over the signature $\Sigma_{+}$of $\mathbf{C P L} \mathbf{L}^{+}$. We then introduce the logic $\mathbf{R m b C}$ which extends $\mathbf{m b C}$ by adding replacement for every formula over $\Sigma$. From the previous observation, it is enough to add replacement for $\neg$ and $\circ$ as new inference rules. Namely: if $\alpha \leftrightarrow \beta$ is a theorem then $\neg \alpha \leftrightarrow \neg \beta$ (is a theorem), and if $\alpha \leftrightarrow \beta$ is a theorem then $\circ \alpha \leftrightarrow \circ \beta$ (is a theorem).

Observe, however, that replacement is in fact a metaproperty (since it states that some formula is a theorem from previous formulas which are assumed to be theorems). It is clear that the two inference rules proposed above for inducing replacement are global instead of local (see Section 6 below): in order to apply each rule, the corresponding premise must be a theorem. This is an analogous situation to the necessitation rule in modal logics. Assuming inference rules of this kind requires changing the definition of derivation from premises in the resulting Hilbert calculus, as we shall see below.

DEFINITION 2.5 The logic $\mathbf{R m b C}$, defined over signature $\Sigma$, is obtained from $\mathbf{m b C}$ by adding the following inference rules:

$$
\frac{\alpha \leftrightarrow \beta}{\neg \alpha \leftrightarrow \neg \beta} \quad\left(\boldsymbol{R}_{\neg}\right) \quad \frac{\alpha \leftrightarrow \beta}{\circ \alpha \leftrightarrow \circ \beta}
$$

\footnotetext{
${ }^{3}$ As usual, $\alpha \leftrightarrow \beta$ is an abbreviation of the formula $(\alpha \rightarrow \beta) \wedge(\beta \rightarrow \alpha)$, and $\gamma[p / \alpha]$ denotes the formula obtained from $\gamma$ by replacing every occurrence of the variable $p$ by the formula $\alpha$.
} 


\section{DeFinition 2.6 (Derivations in RmbC)}

(1) A derivation of a formula $\varphi$ in $\mathbf{R m b C}$ is a finite sequence of formulas $\varphi_{1} \ldots \varphi_{n}$ such that $\varphi_{n}$ is $\varphi$ and, for every $1 \leq i \leq n$, either $\varphi_{i}$ is an instance of an axiom schema of $\mathbf{R m b C}$, or $\varphi_{i}$ is the consequence of some inference rule of $\mathbf{R m b C}$ whose premises appear in the sequence $\varphi_{1} \ldots \varphi_{i-1}$.

(2) We say that a formula $\varphi$ is derivable in $\mathbf{R m b C}$, or that $\varphi$ is a theorem of $\mathbf{R m b C}$, denoted by $\vdash_{\mathbf{R m b C}} \varphi$, if there exists a derivation of $\varphi$ in $\mathbf{R m b C}$.

(3) Let $\Gamma \cup\{\varphi\}$ be a set of formulas over $\Sigma$. We say that $\varphi$ is derivable in $\mathbf{R m b C}$ from the premises $\Gamma$, and we write $\Gamma \vdash_{\mathbf{R m b C}} \varphi$, if either $\varphi$ is derivable in $\mathbf{R m b C}$, or there exists a finite, non-empty subset $\left\{\gamma_{1}, \ldots, \gamma_{n}\right\}$ of $\Gamma$ such that the formula $\left(\gamma_{1} \wedge\left(\gamma_{2} \wedge\left(\ldots \wedge\left(\gamma_{n-1} \wedge \gamma_{n}\right) \ldots\right)\right)\right) \rightarrow \varphi$ is derivable in $\mathbf{R m b C}$.

\section{REMARK 2.7}

(1) The order in which we take the conjunction of elements in the set $\left\{\gamma_{1}, \ldots, \gamma_{n}\right\}$ in item (3) above is immaterial because of the replacement property. In the sequel, we will write $\left(\gamma_{1} \wedge \ldots \wedge \gamma_{n}\right)$ instead of $\left(\gamma_{1} \wedge\left(\gamma_{2} \wedge\left(\ldots \wedge\left(\gamma_{n-1} \wedge \gamma_{n}\right) \ldots\right)\right)\right)$.

(2) From the previous definition, it follows that $\emptyset \vdash_{\mathbf{R m b C}} \varphi$ iff $\vdash_{\mathbf{R m b C}} \varphi$.

(3) Recall that a consequence relation $\vdash$ is said to be Tarskian and finitary if it satisfies the following properties: (i) $\Gamma \vdash \alpha$ whenever $\alpha \in \Gamma$; (ii) if $\Gamma \vdash \alpha$ and $\Gamma \subseteq \Delta$ then $\Delta \vdash \alpha$; (iii) if $\Gamma \vdash \Delta$ and $\Delta \vdash \alpha$ then $\Gamma \vdash \alpha$, where $\Gamma \vdash \Delta$ means that $\Gamma \vdash \delta$ for every $\delta \in \Delta$; and (iv) $\Gamma \vdash \alpha$ implies that $\Gamma_{0} \vdash \alpha$ for some finite $\Gamma_{0}$ contained in $\Gamma$. It is easily seen that the consequence relation $\vdash_{\mathbf{R m b C}}$ given in Definition 2.6(2) is Tarskian and finitary, by virtue of the properties of $\rightarrow$ and $\wedge$ in $\mathbf{R m b C}$ inherited from $\mathbf{C} \mathbf{P} \mathbf{L}^{+}$.

TheOREM 2.8 Let $\mathbf{L}$ be the logic $\mathbf{R m b C}$ or any axiomatic extension of it over the signature $\Sigma$, in which the derivation from premises is defined as in $\mathbf{R} \mathbf{m b C}$. Then, $\mathbf{L}$ satisfies the replacement property.

Proof. Using the properties of $\mathbf{C P L}^{+}$, we have that

$$
\left(\left(\alpha \leftrightarrow \alpha^{\prime}\right) \wedge\left(\beta \leftrightarrow \beta^{\prime}\right)\right) \rightarrow\left((\alpha \# \beta) \leftrightarrow\left(\alpha^{\prime} \# \beta^{\prime}\right)\right)
$$

is a theorem of $\mathbf{L}$, for $\# \in\{\wedge, \vee, \rightarrow\}$. From this, $(\alpha \# \beta) \leftrightarrow\left(\alpha^{\prime} \# \beta^{\prime}\right)$ is a theorem of $\mathbf{L}$ provided that $\left(\alpha \leftrightarrow \alpha^{\prime}\right)$ and $\left(\beta \leftrightarrow \beta^{\prime}\right)$ are theorems of $\mathbf{L}$. On the other hand, the rules $\left(\mathbf{R}_{\neg}\right)$ and $\left(\mathbf{R}_{\circ}\right)$ guarantee that $\left(\# \alpha \leftrightarrow \# \alpha^{\prime}\right)$ is a theorem of $\mathbf{L}$ provided that $\left(\alpha \leftrightarrow \alpha^{\prime}\right)$ is a theorem of $\mathbf{L}$, for $\# \in\{\neg, \circ\}$. From this, it is straightforward to prove, by induction on the complexity of the formula $\gamma(p)]^{4}$ that $\mathbf{L}$ satisfies replacement: If $\alpha \leftrightarrow \beta$ is a theorem of $\mathbf{L}$ then $\gamma[p / \alpha] \leftrightarrow \gamma[p / \beta]$ is a theorem of $\mathbf{L}$. The details are left to the reader.

By considering once again the properties of $\wedge$ and $\rightarrow$ inherited from $\mathbf{C P L}^{+}$, and by the notion of derivation in $\mathbf{R m b C}$, it is easy to see that the Deduction Metatheorem holds in RmbC:

TheOREM 2.9 (Deduction Metatheorem for RmbC)

$\Gamma, \varphi \vdash_{\mathbf{R m b C}} \psi$ if and only if $\Gamma \vdash_{\mathbf{R m b C}} \varphi \rightarrow \psi$.

An algebraic semantics for $\mathbf{R m b C}$ will be given by means of a suitable class of Boolean algebras extended with additional unary operations, which interpret the non-classical connectives. We term these additional operations: LFI operators. In view of the definition of derivations in $\mathbf{R m b C}$ discussed above, the semantic consequence relation will be degree preserving instead of truth preserving (Bou et al., 2009, see). By analogy to modal logics, we may call such a semantics local instead of global. We will return to this point in Section 6 .

\footnotetext{
${ }^{4}$ By convenience, and as is usually done in LFIs (see, e.g., Carnielli and Coniglio (2016a)), the complexity of $\circ \gamma$ is taken to be stricty greater than the complexity of $\neg \gamma$.
} 
Definition 2.10 (BALFIs) $A$ Boolean algebra with LFI operators (BALFI, for short) is an algebra $\mathcal{B}=\langle A, \wedge, \vee, \rightarrow, \neg, \circ, 0,1\rangle$ over $\Sigma_{e}$ such that its reduct $\mathcal{A}=\langle A, \wedge, \vee, \rightarrow, 0,1\rangle$ to $\Sigma_{B A}$ is a Boolean algebra and the unary operators $\neg$ and $\circ$ satisfy: $a \vee \neg a=1$ and $a \wedge \neg a \wedge \circ a=0$, for every $a \in A$. The variety $y^{5}$ of BALFIs will be denoted by $\mathbb{B I}$.

\section{REMARK 2.11}

(1) The set $A$ is called the carrier or domain of $\mathcal{B}$ (and also of $\mathcal{A}$ ).

(2) Recall that an implicative lattice is an algebra $\mathcal{A}=\langle A, \wedge, \vee, \rightarrow, 1\rangle$ where the reduct $\langle A, \wedge, \vee, 1\rangle$ is a lattice with top element 1 in which $\bigvee\{c \in A: a \wedge c \leq b\}$ exists for every $a, b \in A$, and $a \rightarrow b \stackrel{\text { def }}{=} \bigvee\{c \in A: a \wedge c \leq b\}$ for every $a, b \in A \sqrt{6}$ Implicative lattices are in fact distributive. A Heyting algebra is an implicative lattice with least element 0 , where the pseudo-complement is defined as $\sim a \stackrel{\text { def }}{=} a \rightarrow 0$ (see, e.g., Dunn and Hardegree, 2001, 11.2 and 11.3). A Boolean algebra is a Heyting algebra such that $a \vee \sim a=1$ for every $a$. This justifies the use of the signature $\Sigma_{B A}$ for presenting Boolean algebras.

(3) BALFIs are in a sense analogous to the Boolean algebras with operators (BAOs) used as semantics for modal logics (see, e.g., Jónsson (1993)). Observe, however, that LFI operators do not generally distribute over meets or joins.

Definition 2.12 (Degree-preserving BALFI semantics)

(1) A valuation over a BALFI $\mathcal{B}$ is a homomorphism $v:$ For $(\Sigma) \rightarrow \mathcal{B}$ of algebras over $\Sigma$ (where $\mathcal{B}$ is considered, in particular, as an algebra over $\Sigma)$.

(2) Let $\varphi$ be a formula in $\operatorname{For}(\Sigma)$. We say that $\varphi$ is valid in $\mathbb{B I}$, denoted by $\models_{\mathbb{B I}} \varphi$, if, for every $B A L F I \mathcal{B}$ and every valuation $v$ over it, $v(\varphi)=1$.

(3) Let $\Gamma \cup\{\varphi\}$ be a set of formulas in $\operatorname{For}(\Sigma)$. We say that $\varphi$ is a local (or degree-preserving) consequence of $\Gamma$ in $\mathbb{B I}$, denoted by $\Gamma \models_{\mathbb{B I}} \varphi$, if either $\varphi$ is valid in $\mathbb{B I}$, or there exists a finite, non-empty subset $\left\{\gamma_{1}, \ldots, \gamma_{n}\right\}$ of $\Gamma$ such that, for every BALFI $\mathcal{B}$ and every valuation $v$ over it, $\bigwedge_{i=1}^{n} v\left(\gamma_{i}\right) \leq v(\varphi)$ (recalling that, in any Boolean algebra $\mathcal{A}$, the order is given by: $a \leq b$ iff $a \rightarrow b=1)$.

\section{REMARK 2.13}

(1) Defining BALFI valuations as homomorphisms in this way means that $v(\# \varphi)=\# v(\varphi)$, for $\# \in\{\neg, \circ\}$, and $v(\varphi \# \psi)=v(\varphi) \# v(\psi)$, for $\# \in\{\wedge, \vee, \rightarrow\}$.

(2) Note that $\Gamma \models_{\mathbb{B I}} \varphi$ iff either $\varphi$ is valid in $\mathbb{B I}$, or there exists a finite, non-empty subset $\left\{\gamma_{1}, \ldots, \gamma_{n}\right\}$ of $\Gamma$ such that $\left(\gamma_{1} \wedge \ldots \wedge \gamma_{n}\right) \rightarrow \varphi$ is valid. This follows easily from the definitions.

TheOREM 2.14 (Soundness of RmbC w.r.t. $\mathbb{B I}$ )

Let $\Gamma \cup\{\varphi\} \subseteq \operatorname{For}(\Sigma)$. Then: $\Gamma \vdash_{\mathbf{R m b C}} \varphi$ implies that $\Gamma \models_{\mathbb{B I}} \varphi$.

Proof. Let $\varphi$ be a an instance of an axiom of $\mathbf{R m b C}$. It is immediate to see that, for every $\mathcal{B}$ and every valuation $v$ on it, $v(\varphi)=1$. Now, let $\alpha, \beta \in \operatorname{For}(\Sigma)$. If $v(\alpha \rightarrow \beta)=1$ and $v(\alpha)=1$ then, since $v(\alpha \rightarrow \beta)=v(\alpha) \rightarrow v(\beta)$, it follows that $v(\beta)=1$. On the other hand, if $v(\alpha \leftrightarrow \beta)=1$ then $v(\alpha)=v(\beta)$ and so $v(\# \alpha)=\# v(\alpha)=\# v(\beta)=v(\# \beta)$, hence it follows that $v(\# \alpha \leftrightarrow \# \beta)=1$ for $\# \in\{\neg, \circ\}$. From this, by induction on the length of a derivation of $\varphi$ in $\mathbf{R m b C}$, it can be easily proven that $\varphi$ is valid in $\mathbb{B I}$ whenever $\varphi$ is derivable in $\mathbf{R m b C}$. Now, suppose that $\Gamma \vdash_{\mathbf{R m b C}} \varphi$. If $\vdash_{\mathbf{R m b C}} \varphi$ then, by the observation above, $\varphi$ is valid in $\mathbb{B I}$ and so $\Gamma \models \mathbb{B I} \varphi$. On the other hand, if there exists a finite, non-empty subset $\left\{\gamma_{1}, \ldots, \gamma_{n}\right\}$ of $\Gamma$ such that $\vdash_{\mathbf{R m b C}}\left(\gamma_{1} \wedge \ldots \wedge \gamma_{n}\right) \rightarrow \varphi$ then, once again by the observation above, $\mid=\mathbb{B I}\left(\gamma_{1} \wedge \ldots \wedge \gamma_{n}\right) \rightarrow \varphi$. This shows that $\Gamma=_{\mathbb{B I}} \varphi$, by Remark 2.13 .

\footnotetext{
${ }^{5}$ Recall that a class of algebras is a variety if it can be axiomatized by means of equations.

${ }^{6}$ Here $\leq$ is the partial order associated to the lattice, namely: $a \leq b$ iff $a=a \wedge b$ iff $b=a \vee b$, and $\bigvee X$ denotes the supremum of the set $X \subseteq A$ w.r.t. $\leq$, provided that it exists. Observe that $a \leq b$ iff $a \rightarrow b=1$.
} 
TheOREM 2.15 (Completeness of RmbC w.r.t. $\mathbb{B I}$ )

Let $\Gamma \cup\{\varphi\} \subseteq$ For $(\Sigma)$. Then: $\Gamma \models_{\mathbb{B I}} \varphi$ implies that $\Gamma \vdash_{\mathbf{R m b C}} \varphi$.

Proof. Define the following relation on $\operatorname{For}(\Sigma): \alpha \equiv \beta$ iff $\vdash_{\mathbf{R m b C}} \alpha \leftrightarrow \beta$. It is clearly an equivalence relation, by the properties of $\mathbf{C P L}^{+}$. Let $A_{\text {can }} \stackrel{\text { def }}{=} \operatorname{For}(\Sigma) / \equiv$ be the quotient set, and define over $A_{c a n}$ the following operations: $[\alpha] \#[\beta] \stackrel{\text { def }}{=}[\alpha \# \beta]$, for $\# \in\{\wedge, \vee, \rightarrow\}$, where $[\alpha]$ denotes the equivalence class of $\alpha$ w.r.t. $\equiv$. Let $0 \stackrel{\text { def }}{=}[\alpha \wedge \neg \alpha \wedge \circ \alpha]$ and $1 \stackrel{\text { def }}{=}[\alpha \vee \neg \alpha]$. These operations and constants are clearly well-defined, by Theorem 2.8, and so they induce a structure of Boolean algebra over the set $A_{\text {can }}$, which will be denoted by $\mathcal{A}_{\text {can }}$. Let $\mathcal{B}_{\text {can }}$ be its expansion to $\Sigma_{e}$ by defining $\#[\alpha] \stackrel{\text { def }}{=}[\# \alpha]$, for $\# \in\{\neg, \circ\}$. By Theorem 2.8 these operations are well-defined, and it is immediate to see that $\mathcal{B}_{\text {can }}$ is a BALFI. Let $v_{\text {can }}: \operatorname{For}(\Sigma) \rightarrow \mathcal{B}_{\text {can }}$ given by $v_{\text {can }}(\alpha)=[\alpha]$. Clearly $v_{\text {can }}$ is a valuation over $\mathcal{B}_{\text {can }}$ such that $v_{\text {can }}(\alpha)=1$ iff $\vdash_{\mathbf{R m b C}} \alpha$.

Now, suppose that $\Gamma \models_{\mathbb{B I}} \varphi$, and recall Remark 2.13 . If $\models_{\mathbb{B I}} \varphi$ then, in particular, $v_{\text {can }}(\varphi)=1$ and so $\vdash_{\mathbf{R m b C}} \varphi$. From this, $\Gamma \vdash_{\mathbf{R m b C}} \varphi$. On the other hand, if there exists a finite, non-empty subset $\left\{\gamma_{1}, \ldots, \gamma_{n}\right\}$ of $\Gamma$ such that $==_{\mathbb{B I}}\left(\gamma_{1} \wedge \ldots \wedge \gamma_{n}\right) \rightarrow \varphi$ then, in particular, $v_{\text {can }}\left(\left(\gamma_{1} \wedge \ldots \wedge \gamma_{n}\right) \rightarrow\right.$ $\varphi)=1$. This means that $\vdash_{\mathbf{R m b C}}\left(\gamma_{1} \wedge \ldots \wedge \gamma_{n}\right) \rightarrow \varphi$ and so $\Gamma \vdash_{\mathbf{R m b C}} \varphi$.

Definition 2.16 The pair $\left\langle\mathcal{B}_{c a n}, v_{c a n}\right\rangle$ defined in the proof of Theorem 2.15 is called the canonical model of $\mathbf{R m b C}$.

EXAMPLE 2.17 (BALFIs over $\left.\wp\left(\left\{w_{1}, w_{2}\right\}\right)\right)$ Let $\mathcal{A}_{4}=\wp\left(\left\{w_{1}, w_{2}\right\}\right)=\{0, a, b, 1\}$ be the powerset of $W_{2}=\left\{w_{1}, w_{2}\right\}$ such that $0=\emptyset, a=\left\{w_{1}\right\}, b=\left\{w_{2}\right\}$ and $1=\left\{w_{1}, w_{2}\right\}$. Then, the BALFIs defined by expanding the Boolean algebra $\mathcal{A}_{4}$ are shown in Figure 1.

\begin{tabular}{|c|c|c|}
\hline$z$ & $\neg z$ & oz \\
\hline \hline 1 & $0|a| b \mid 1$ & $x \mid(0$ or $b) \mid(0$ or $a) \mid 0$ \\
\hline$a$ & $b \mid 1$ & $x \mid(0$ or $b)$ \\
\hline$b$ & $a \mid 1$ & $x \mid(0$ or $a)$ \\
\hline 0 & 1 & $x$ \\
\hline
\end{tabular}

Figure 1: Set of BALFIs over $\mathcal{A}_{4}$ (the symbol | separates the possible options for the values of $\neg z$ and $\circ z$ for every value of $z$, while $x$ stands for any element of $\mathcal{A}_{4}$ )

On each row of Figure 1, each choice in the ith position of the sequence of options in the column for $\neg z$ forces a choice in the ith position of the sequence of options in the column for $\circ z$. For instance, if in the current BALFI we choose $\neg 1=b$ then there are two possibilities for the value of $\circ 1$ in that BALFI: either $\circ 1=0$ or $\circ 1=a$. On the other hand, by choosing that $\neg a=1$ it forces that either $\circ a=0$ or $\circ a=b$. Otherwise, if $\neg a=b$ then $\circ$ a can be arbitrarily chosen.

REMARK 2.18 Observe that the rules $\left(\boldsymbol{R}_{\neg}\right)$ and $\left(\boldsymbol{R}_{\circ}\right)$ do not ensure that $\vdash_{\mathbf{R m b C}}(\alpha \leftrightarrow \beta) \rightarrow$ $(\neg \alpha \leftrightarrow \neg \beta)$ and $\vdash_{\mathbf{R m b C}}(\alpha \leftrightarrow \beta) \rightarrow(\circ \alpha \leftrightarrow \circ \beta)$ in general. Consider, for instance $\alpha=p$ and $\beta=q$ where $p$ and $q$ are two different propositional variables, and take the BALFI $\mathcal{B}$ (in Figure 2) defined over the Boolean algebra $\wp\left(\left\{w_{1}, w_{2}\right\}\right)$, according to Example 2.17.

Now, consider a valuation $v$ over $\mathcal{B}$ such that $v(p)=a$ and $v(q)=1$. Hence $v(\neg p)=b$, $v(\neg q)=1, v(\circ p)=a$ and $v(\circ q)=0$. From this $v(p \leftrightarrow q)=a$ and $v(\neg p \leftrightarrow \neg q)=v(\circ p \leftrightarrow \circ q)=b$. 


\begin{tabular}{|c|c|c|}
\hline$z$ & $\neg z$ & $\circ z$ \\
\hline \hline 1 & 1 & 0 \\
\hline$a$ & $b$ & $a$ \\
\hline$b$ & 1 & $a$ \\
\hline 0 & 1 & $b$ \\
\hline
\end{tabular}

Figure 2: Counter-model BALFI (over $\left.\mathcal{A}_{4}\right)$ for $\vdash_{\mathbf{R m b C}}(\alpha \leftrightarrow \beta) \rightarrow(\# \alpha \leftrightarrow \# \beta) ; \# \in\{\neg, \circ\}$

Therefore $v((p \leftrightarrow q) \rightarrow(\neg p \leftrightarrow \neg q))=v((p \leftrightarrow q) \rightarrow(\circ p \leftrightarrow \circ q))=b$. That is, none of the last two formulas is valid in $\mathbf{R m b C}$. Of course both formulas hold if $\vdash_{\mathbf{R m b C}}(\alpha \leftrightarrow \beta)$, by $\left(\boldsymbol{R}_{\neg}\right)$ and $\left(\boldsymbol{R}_{\circ}\right)$.

Evidently, $\mathbf{R m b C}$ is paraconsistent: in the BALFI $\mathcal{B}$ we just defined above, the given valuation $v$ shows that $q, \neg q \nvdash_{\mathbf{R m b C}} p$. Now, recalling Definition 2.1, RmbC is clearly an LFI. Consider the following BALFI $\mathcal{B}^{\prime}$ (in Figure 3) defined over $\wp\left(\left\{w_{1}, w_{2}\right\}\right)$, using again Example 2.17.

\begin{tabular}{|c|c|c|}
\hline$z$ & $\neg z$ & $\circ z$ \\
\hline \hline 1 & 0 & 1 \\
\hline$a$ & 1 & 0 \\
\hline$b$ & 1 & 0 \\
\hline 0 & 1 & 1 \\
\hline
\end{tabular}

Figure 3: BALFI (over $\mathcal{A}_{4}$ ) validating the principle of gentle explosion in a non-trivial way

Take a valuation $v^{\prime}$ over $\mathcal{B}^{\prime}$ such that $v^{\prime}(p)=1$ and $v^{\prime}(q)=a$. This shows that $p, o p \nvdash_{\mathbf{R m b C}} q$. Now, a valuation $v^{\prime \prime}$ over $\mathcal{B}^{\prime}$ such that $v^{\prime \prime}(p)=0$ and $v^{\prime \prime}(q)=b$ shows that $\neg p, \circ p \nvdash_{\mathbf{R m b C}} q$. On the other hand, from axiom (bc1), the axioms of $\mathbf{C P L}^{+}$, and by Definition 2.5(3), it is the case that $\alpha, \neg \alpha, \circ \alpha \vdash_{\mathbf{R m b C}} \beta$ for any formulas $\alpha$ and $\beta$.

\section{Adding replacement to extensions of mbC: A solution to an open problem}

In Carnielli and Marcos (2002), the first study on LFIs, the replacement property was analyzed under the name (IpE), presented in the following (equivalent) way:

(IpE) if $\alpha_{i} \dashv \vdash \beta_{i}($ for $1 \leq i \leq n)$ then $\varphi\left(\alpha_{1}, \ldots, \alpha_{n}\right) \dashv \varphi\left(\beta_{1}, \ldots, \beta_{n}\right)$

for any formulas $\alpha_{i}, \beta_{i}, \varphi$. In that paper an important question was posed: to define extensions of $\mathbf{b C}$ and $\mathbf{C i}$ (two axiomatic extensions of $\mathbf{m b C}$ to be analyzed below) which satisfy (IpE) while still being paraconsistent. 7 In this section, we will present a general (in the sense of minimal) solution to that open problem, obtained by extending axiomatically $\mathbf{R m b C}$. In what follows,

\footnotetext{
${ }^{7}$ In Carnielli and Marcos (2002), page 41, one reads: "The question then would be if (IpE) could be obtained for real LFIs". On page 54, after observing that in extensions of Ci it is enough ensuring (IpE) for $\neg$, since it implies (IpE) for $\circ$, it is said that "We suspect that this can be done, but we shall leave it as an open problem at this point". Finally, they observe on page 55, footnote 17 that certain 8-valued matrices presented by Urbas satisfy (IpE) for an extension of bC. However, this logic is not paraconsistent. After this, they claim that "the question is still left open as to whether there are paraconsistent such extensions of bC!".
} 
some LFIs which are axiomatic extensions of $\mathbf{m b C}$ (bC and $\mathbf{C i}$, among others) will be enriched with replacement, in a similar way as we did for $\mathbf{R m b C}$.

Definition 3.1 (Some extensions of $\mathbf{m b C}$ ) Consider the following axioms presented in Carnielli and Marcos (2002) and Carnielli and Coniglio (2016a):

$$
\begin{gathered}
\circ \alpha \vee(\alpha \wedge \neg \alpha) \\
\neg \circ \alpha \rightarrow(\alpha \wedge \neg \alpha) \\
\neg(\alpha \wedge \neg \alpha) \rightarrow \circ \alpha \\
\neg \neg \alpha \rightarrow \alpha \\
\alpha \rightarrow \neg \neg \alpha \\
(\circ \alpha \wedge \circ \beta) \rightarrow \circ(\alpha \wedge \beta) \\
(\circ \alpha \wedge \circ \beta) \rightarrow \circ(\alpha \vee \beta) \\
(\circ \alpha \wedge \circ \beta) \rightarrow \circ(\alpha \rightarrow \beta)
\end{gathered}
$$

\section{REMARK 3.2}

(1) Axiom (ciw) was introduced by Avron (2005) by means of two axioms, (k1): $\circ \alpha \vee \alpha$ and $(\mathbf{k} 2): \circ \alpha \vee \neg \alpha$. Strictly speaking, (k1) and (k2) were presented as rules in a standard Gentzen calculus.

(2) A classical negation (denoted by $\sim$ ) is definable in $\mathbf{~ m b C ~ a s ~} \sim \alpha=\alpha \rightarrow \perp$. Here, $\perp$ denotes any formula of the form $\beta \wedge \neg \beta \wedge \circ \beta$. It is not difficult to show that $\circ \alpha \rightarrow \sim(\alpha \wedge \neg \alpha)$ is a theorem of $\mathbf{~ m b C}$, and thus of $\mathbf{R m b C}$. Also notice that the converse of this formula, namely, $\sim(\alpha \wedge \neg \alpha) \rightarrow \circ \alpha$, is derived in $\mathbf{m b C}$ (hence, in $\mathbf{R m b C}$ ) from (ciw).

(3) It can also be shown that $(\alpha \wedge \neg \alpha) \rightarrow \neg \circ \alpha$, and $\circ \alpha \rightarrow \neg(\alpha \wedge \neg \alpha)$ are theorems of $\mathbf{m b C}$ and therefore of $\mathbf{R m b C}$ (for instance, by applying the Deduction Metatheorem to Theorem 49 (ii) and (iii) in Carnielli et al. (2007)). Notice that these formulas are the 'converses' of (ci) and (cl) respectively.

Definition 3.3 Let $\mathcal{B}=\langle A, \wedge, \vee, \rightarrow, \neg, \circ, 0,1\rangle$ be a BALFI, and let $\varphi$ be a formula over $\Sigma$. $W e$ say that $\mathcal{B}$ is a model of $\varphi$ (considered as an axiom schema), denoted by $\mathcal{B} \Vdash \varphi$, if $v(\varphi)=1$ for every valuation $v$ over $\mathcal{B}$.

As mentioned in Remark 2.11 the Boolean complement in Boolean algebras defined over the signature $\Sigma_{B A}$ is given by $\sim a=a \rightarrow 0$.

Proposition 3.4 Let $\mathcal{B}=\langle A, \wedge, \vee, \rightarrow, \neg, \circ, 0,1\rangle$ be a BALFI. Then:

(1) $\mathcal{B}$ is a model of (ciw) iff $\mathcal{B}$ satisfies the equation $\circ a=\sim(a \wedge \neg a)$ for every $a \in A$;

(2) $\mathcal{B}$ is a model of (ci) iff $\mathcal{B}$ satisfies the equation $\neg \circ a=a \wedge \neg a$ for every $a \in A$;

(3) $\mathcal{B}$ is a model of $(\mathbf{c l})$ iff $\mathcal{B}$ satisfies the equation $\circ a=\neg(a \wedge \neg a)$ for every $a \in A$;

(4) $\mathcal{B}$ is a model of $(\mathbf{c f})$ iff $\mathcal{B}$ satisfies the equation $a \wedge \neg \neg a=\neg \neg$ for every $a \in A$;

(5) $\mathcal{B}$ is a model of (ce) iff $\mathcal{B}$ satisfies the equation $a \wedge \neg \neg a=$ a for every $a \in A$;

(6) $\mathcal{B}$ is a model of $\left(\mathbf{c a}_{\#}\right)$ iff $\mathcal{B}$ satisfies the equation $(\circ a \wedge \circ b) \wedge \circ(a \# b)=\circ a \wedge \circ b$ for every $a, b \in A$, for each $\# \in\{\wedge, \vee, \rightarrow\}$.

Proof. We prove (1). Suppose that $\mathcal{B}$ is a model of (ciw). Then, $v(\circ p \vee(p \wedge \neg p))=\circ v(p) \vee$ $(v(p) \wedge \neg v(p))=1$ for every variable $p$ and any valuation $v$. Since $v(p)$ can take an arbitrary value in $A$, this is equivalent to say that $\circ a \vee(a \wedge \neg a)=1$ for every $a \in A$. Since $\circ a \wedge(a \wedge \neg a)=0$ (by definition of BALFIs), it follows that $\circ a=\sim(a \wedge \neg a)$. The converse is immediate. Proofs for (2) and (3) are obtained in an analogous way (recalling Remark 3.2 (3)). The proofs of (4), (5), and (6) are immediate from the definitions. 
Let $A x$ be a set formed by one or more of the axiom schemas introduced in Definition 3.1, and let $\mathbf{m b C}(A x)$ be the logic defined by the Hilbert calculus obtained from $\mathbf{m b C}$ by adding the set $A x$ of axiom schemas. Let $\mathbb{B I}(A x)$ be the class of BALFIs which are models of every axiom in $A x$. Clearly, $\mathbb{B I}(A x)$ is a variety of algebras, since it is characterized by a set of equations. Finally, let $\mathbf{R m b C}(A x)$ be the logic obtained from $\mathbf{R m b C}$ by adding the set $A x$ of axiom schemas. It is simple to adapt the proofs of Theorems 2.14 and 2.15 (in particular, by defining for each logic the corresponding canonical model, as in Definition 2.16) in order to obtain the following:

Theorem 3.5 (Soundness and completeness of $\mathbf{R m b C}(A x)$ w.r.t. $\mathbb{B I}(A x)$ ) Let $\Gamma \cup\{\varphi\} \subseteq$ For $(\Sigma)$. Then: $\Gamma \vdash_{\mathbf{R m b C}(A x)} \varphi$ if and only if $\Gamma \models_{\mathbb{B I}(A x)} \varphi$.

From this important result, some properties of the calculi $\mathbf{R m b C}(A x)$ can be easily proven by algebraic methods, that is, by means of BALFIs. For instance:

Proposition $3.6 \mathbb{B I}(\{\mathbf{c i}, \mathbf{c f}\})=\mathbb{B} \mathbb{I}(\{\mathbf{c l}, \mathbf{c f}\})=\mathbb{B I}(\{\mathbf{c i}, \mathbf{c l}, \mathbf{c f}\})$. Hence, the logics $\mathbf{R m b C}(\{\mathbf{c i}, \mathbf{c f}\})$, $\mathbf{R m b C}(\{\mathbf{c l}, \mathbf{c f}\})$ and $\mathbf{R m b C}(\{\mathbf{c i}, \mathbf{c l}, \mathbf{c f}\})$ coincide.

Proof. Recall from Remark 3.2 (3) that $\vdash_{\mathbf{R m b C}}(\alpha \wedge \neg \alpha) \rightarrow \neg \circ \alpha$ and $\vdash_{\mathbf{R m b C}} \circ \alpha \rightarrow \neg(\alpha \wedge \neg \alpha)$. Then, for every BALFI $\mathcal{B}$ with carrier $A$, and every $a \in A,(a \wedge \neg a) \leq \neg \circ a$ and $\circ a \leq \neg(a \wedge \neg a)$. Let $\mathcal{B} \in \mathbb{B I}(\{\mathbf{c i}, \mathbf{c f}\})$, and let $a \in A$. Then $a \wedge \neg a=\neg \circ a$ and so $\neg(a \wedge \neg a)=\neg \neg \circ a \leq \circ a$. Therefore $\mathcal{B} \in \mathbb{B I}(\{\mathbf{c l}, \mathbf{c f}\})$. Conversely, suppose that $\mathcal{B} \in \mathbb{B I}(\{\mathbf{c l}, \mathbf{c f}\})$ and let $a \in A$. Since $\circ a=\neg(a \wedge \neg a)$ then $\neg \circ a=\neg \neg(a \wedge \neg a) \leq(a \wedge \neg a)$. From this, $\mathcal{B} \in \mathbb{B} \mathbb{I}(\{\mathbf{c i}, \mathbf{c f}\})$. This shows the first part of the Proposition. The second part follows from Theorem 3.5 .

EXAMPle 3.7 (BALFIs for RmbCciw) The logic $\mathbf{m b C}$ (ciw) was considered in Carnielli and Coniglio (2016a) under the name mbCciw. This logic was introduced in Avron (2005) under the name $\mathbf{B}[\{(\mathbf{k} \mathbf{1}),(\mathbf{k} \mathbf{2})\}]$, presented by means of a standard Gentzen calculus such that $\mathbf{B}$ is a Gentzen calculus for $\mathbf{m b C}$. The logic $\mathbf{m b C c i w}$ is the least extension of $\mathbf{m b C}$ in which the consistency connective can be defined in terms of the other connectives, namely: $\circ \alpha$ is equivalent to $\sim(\alpha \wedge \neg \alpha)$ \& Let $\mathbf{R m b C c i w}$ be the logic $\mathbf{R m b C}(\mathbf{c i w})$. Because of the satisfaction of the replacement property, and given that the consistency connective can be defined in terms of the other connectives, the connective o can be eliminated from the signature, and so we consider the logic $\mathbf{R m b C c i w}$ defined over the signature $\Sigma_{C_{0}}$ (recall Definition 2.2), obtained from $\mathbf{C P L}^{+}$by adding $(\boldsymbol{A x 1 0}),\left(\boldsymbol{R}_{\neg}\right)$, and axiom schema $(\mathbf{B o t}): \overline{0} \rightarrow \alpha$. In such presentation of $\mathbf{R m b C c i w ,}$ the strong negation is defined by the formula $\sim \alpha=\alpha \rightarrow \overline{0}$. The algebraic models for this presentation of $\mathbf{R m b C c i w}$ are given by BALFIs $\mathcal{B}=\langle A, \wedge, \vee, \rightarrow, \neg, 0,1\rangle$ over $\Sigma_{C_{e}}$ such that its reduct $\mathcal{A}=\langle A, \wedge, \vee, \rightarrow, 0,1\rangle$ to $\Sigma_{B A}$ is a Boolean algebra and the unary operator $\neg$ satisfies $a \vee \neg a=1$ for every $a \in A$. On the other hand, the term $\circ a$ is an abbreviation for $\sim(a \wedge \neg a)$ in such BALFIs.

It is also interesting to observe that o satisfies a sort of necessitation rule (by analogy to modal logics) in certain extensions of $\mathbf{R m b C}$ :

Proposition 3.8 Consider the necessitation rule for $\circ$ :

$$
\frac{\alpha}{\circ \alpha} \quad\left(N e c_{\circ}\right)
$$

Then, $\left(N e c_{\circ}\right)$ is an admissible rule in $\mathbf{R m b C}(\{\mathbf{c l}, \mathbf{c e}\}) 9^{9}$

\footnotetext{
${ }^{8}$ Recall from Remark 3.2 (2) that $\sim$ denotes the classical negation definable in mbC as $\sim \alpha=\alpha \rightarrow \perp$, where $\perp$ denotes any formula of the form $\beta \wedge \neg \beta \wedge \circ \beta$. Thus, rigorously speaking, $\circ$ is not defined in terms of the other connectives, since $\circ$ is essential on order to define $\perp$. So, the right signature for mbCciw and its extensions is $\Sigma_{C_{0}}$, introduced in Definition 2.2

${ }^{9}$ Recall that a structural inference rule is admissible in a logic $\mathbf{L}$ if the following holds: whenever the premises of an instance of the rule are theorems of $\mathbf{L}$, then the conclusion of the same instance of the rule is a theorem of L.
} 
Proof. Assume that $\vdash_{\mathbf{R m b C}(\{\mathbf{c l}, \mathbf{c e}\})} \alpha$. By the rules of $\mathbf{C P L}^{+}$it follows that $\vdash_{\mathbf{R m b C}(\{\mathbf{c l}, \mathbf{c e}\})}$ $\beta \leftrightarrow(\alpha \wedge \beta)$ for every formula $\beta$. In particular, $\vdash_{\mathbf{R m b C}(\{\mathbf{c l}, \mathbf{c e}\})} \neg \alpha \leftrightarrow(\alpha \wedge \neg \alpha)$ and so, by $\left(\mathbf{R}_{\neg}\right)$, $\vdash_{\mathbf{R m b C}(\{\mathbf{c l}, \mathbf{c e}\})} \neg \neg \alpha \leftrightarrow \neg(\alpha \wedge \neg \alpha)$. On the other hand, from $\vdash_{\mathbf{R m b C}(\{\mathbf{c l}, \mathbf{c e}\})} \alpha$ it follows that $\vdash_{\mathbf{R m b C}(\{\mathbf{c l}, \mathbf{c e}\})} \neg \neg \alpha$, by (ce) and (MP). Then $\vdash_{\mathbf{R m b C}(\{\mathbf{c l}, \mathbf{c e}\})} \neg(\alpha \wedge \neg \alpha)$. By (cl) and (MP) we conclude that $\vdash_{\mathbf{R m b C}(\{\mathbf{c l}, \mathbf{c e}\})} \circ \alpha$.

We can now provide a solution to the first open problem posed in Carnielli and Marcos (2002):

EXAMPLE 3.9 (A paraconsistent extension of $\mathbf{b C}$ with replacement)

Consider the logic $\mathbf{b C}$ introduced in Carnielli and Marcos (2002). By using the notation proposed above, $\mathbf{b C}$ corresponds to $\mathbf{m b C}(\mathbf{c f}){ }^{10}$ Then $\mathbf{R b C}$ (that is, $\left.\mathbf{R m b C}(\mathbf{c f})\right)$ is an extension of $\mathbf{b C}$ which satisfies replacement (by Theorem 2.8) while being still paraconsistent. Moreover, RbC is an LFI. These facts can be easily proven by using the BALFI $\mathcal{B}^{\prime}$ defined over $\wp\left(\left\{w_{1}, w_{2}\right\}\right)$ considered in Remark 2.18 (see Figure 4).

\begin{tabular}{|c|c|c|}
\hline$z$ & $\neg z$ & $\circ z$ \\
\hline \hline 1 & 0 & 1 \\
\hline$a$ & 1 & 0 \\
\hline$b$ & 1 & 0 \\
\hline 0 & 1 & 1 \\
\hline
\end{tabular}

Figure 4: A 4-elements paraconsistent BALFI for $\mathbf{R b C}$

In fact, it is immediate to see that $\mathcal{B}^{\prime}$ is a model of $(\mathbf{c f})$. It is worth noting that $\mathcal{B}^{\prime}$ is not a model of (ciw): $0=\circ a \neq \sim(a \wedge \neg a)=\sim a=b$. Therefore, $\mathcal{B}^{\prime}$ is neither a model of (ci) nor of (cl), given that any of these axioms implies (ciw). (2002):

We can now offer a solution to the second open problem posed in Carnielli and Marcos

EXAmple 3.10 (A paraconsistent extension of $\mathbf{C i}$ with replacement) Now, consider the logic Ci introduced in Carnielli and Marcos (2002), which corresponds to $\mathbf{m b C}(\{\mathbf{c f}, \mathbf{c i}\})$, and let $\mathbf{R C i}=\mathbf{R m b C}(\{\mathbf{c f}, \mathbf{c i}\})$. By Proposition 3.6, $\mathbf{R C i}$ also derives the schema $(\mathbf{c l})$. It can be proven that $\mathbf{R C i}$ is an extension of $\mathbf{C i}$ which satisfies replacement (by Theorem 2.8) while it is still paraconsistent. In order to prove this, consider the BALFI $\mathcal{B}^{\prime \prime}$ defined over the Boolean algebra $\mathcal{A}_{16}=\wp\left(W_{4}\right)$, the powerset of $W_{4}=\left\{w_{1}, w_{2}, w_{3}, w_{4}\right\}$ displayed in Figure 5 . In that figure, $X$ is different from $\left\{w_{1}, w_{2}\right\}$ and $\left\{w_{3}, w_{4}\right\}$ (note that $0=\emptyset$ and $1=W_{4}$ ). It is immediate to see that $\mathcal{B}^{\prime \prime}$ is a BALFI for RCi. Hence, using this model it follows easily that $\mathbf{R C i}$ is a paraconsistent extension of $\mathbf{C i}$ which satisfies (IpE) and (cl).

Another paraconsistent model for $\mathbf{R C i}$ defined over $\mathcal{A}_{16}$ is displayed in Figure 6 . Here, the cardinal of $X$ is different from $2{ }^{11}$

\footnotetext{
${ }^{10}$ We will write $\operatorname{mbC}(\varphi), \mathbf{R m b C}(\varphi)$ and $\mathbb{B} \mathbb{I}(\varphi)$ instead of $\mathbf{m b C}(\{\varphi\}), \mathbf{R m b C}(\{\varphi\})$ and $\mathbb{B I}(\{\varphi\})$, respectively.

${ }^{11} \mathrm{It}$ is worth noting that many of the experiments leading to the generation of the two models presented here were carried out with the help of the model finder Nitpick (Blanchette and Nipkow, 2010), which is part of the automated tools integrated into the proof assistant Isabelle/HOL (Nipkow et al., 2002); recall also the comments in Footnote 1.
} 


\begin{tabular}{|c|c|c|}
\hline$z$ & $\neg z$ & $\circ z$ \\
\hline \hline$\left\{w_{1}, w_{2}\right\}$ & $\left\{w_{1}, w_{3}, w_{4}\right\}$ & $\left\{w_{2}, w_{3}, w_{4}\right\}$ \\
\hline$\left\{w_{3}, w_{4}\right\}$ & $\left\{w_{1}, w_{2}, w_{3}\right\}$ & $\left\{w_{1}, w_{2}, w_{4}\right\}$ \\
\hline$X$ & $W_{4} \backslash X$ & $W_{4}$ \\
\hline
\end{tabular}

Figure 5: A 16-elements paraconsistent BALFI for $\mathbf{R C i}$

\begin{tabular}{|c|c|c|}
\hline$z$ & $\neg z$ & $\circ z$ \\
\hline \hline$\left\{w_{1}, w_{2}\right\}$ & $\left\{w_{2}, w_{3}, w_{4}\right\}$ & $\left\{w_{1}, w_{3}, w_{4}\right\}$ \\
\hline$\left\{w_{1}, w_{3}\right\}$ & $\left\{w_{2}, w_{3}, w_{4}\right\}$ & $\left\{w_{1}, w_{2}, w_{4}\right\}$ \\
\hline$\left\{w_{1}, w_{4}\right\}$ & $\left\{w_{2}, w_{3}, w_{4}\right\}$ & $\left\{w_{1}, w_{2}, w_{3}\right\}$ \\
\hline$\left\{w_{2}, w_{3}\right\}$ & $\left\{w_{1}, w_{3}, w_{4}\right\}$ & $\left\{w_{1}, w_{2}, w_{4}\right\}$ \\
\hline$\left\{w_{2}, w_{4}\right\}$ & $\left\{w_{1}, w_{3}, w_{4}\right\}$ & $\left\{w_{1}, w_{2}, w_{3}\right\}$ \\
\hline$\left\{w_{3}, w_{4}\right\}$ & $\left\{w_{1}, w_{2}, w_{4}\right\}$ & $\left\{w_{1}, w_{2}, w_{3}\right\}$ \\
\hline$X$ & $W_{4} \backslash X$ & $W_{4}$ \\
\hline
\end{tabular}

Figure 6: Another 16-elements paraconsistent BALFI for RCi

EXAMPLE 3.11 We can offer now a model of $\mathbf{R m b C}(\mathbf{c l})$ that does not satisfy axiom (cf). For this purpose consider the BALFI $\mathcal{B}^{\prime \prime \prime}$ defined over the Boolean algebra $\mathcal{A}_{4}=\wp\left(\left\{w_{1}, w_{2}\right\}\right)=$ $\{0, a, b, 1\}$ according to Example 2.17, and displayed in Figure 7 . Observe that $\mathcal{B}^{\prime \prime \prime} \Vdash$ cl. However, $\mathcal{B}^{\prime \prime \prime}$ is not a model of (cf): $\neg \neg 0=a \not \leq 0$.

\begin{tabular}{|c|c|c|}
\hline$z$ & $\neg z$ & $\circ z$ \\
\hline \hline 1 & $a$ & $b$ \\
\hline$a$ & $b$ & 1 \\
\hline$b$ & $a$ & 1 \\
\hline 0 & 1 & 1 \\
\hline
\end{tabular}

Figure 7: A paraconsistent BALFI model of $\mathbf{R m b C}(\mathbf{c l})$ that does not satisfy axiom (cf)

\section{Limits for replacement while preserving paraconsistency}

In Carnielli and Marcos (2002, Theorem 3.51) some sufficient conditions were given to show that certain extensions of $\mathbf{b} \mathbf{C}$ and $\mathbf{C i}$ cannot satisfy replacement while being still paraconsistent. This result shows that there are limits, much before reaching classical logic CPL, for extending $\mathbf{R m b C}$ while preserving paraconsistency. This result will now be applied in order to obtain two important examples of LFIs that cannot be extended with replacement at risk of losing their character of paraconsistency.

The first example, to begin with, is in fact a family of 8,192 examples:

EXAMPLE 4.1 (Three-valued LFIs)

Recall the family of $8 K b$ three-valued LFIs introduced by Marcos in an unpublished draft, and 
discussed in Carnielli and Marcos (2002, Section 3.11) and in Carnielli et al. (2007, Section 5.3). As it was observed in these references, the schema $\neg(\alpha \wedge \neg \alpha)$ is valid in all of these logics. In addition, all these logics are models of axioms (ci) and (cf), i.e., they extend $\mathbf{C i}$ (see, e.g., Carnielli et al., 2007, Theorem 130). But in Carnielli and Marcos (2002, Theorem 3.51(ii)) it was proved that (IpE) cannot hold in any paraconsistent extension of $\mathbf{C i}$ in which the schema $\neg(\alpha \wedge \neg \alpha)$ is valid. As a consequence, the inference rules $\left(\boldsymbol{R}_{\neg}\right)$ and $\left(\boldsymbol{R}_{\circ}\right)$ cannot be added to any of them at the risk of losing paraconsistency. Indeed, if $\mathbf{L}$ is any of such three-valued logics, the corresponding logic $\mathbf{R L}$ obtained by adding both rules will derive the axiom schema $\circ \alpha$ (the proof of this fact can be easily adapted from the one for Theorem 3.51(ii) presented in Carnielli and Marcos (2002)). But then, the negation $\neg$ is explosive in $\mathbf{R L}$, by (bc1) and (MP). This shows that these three-valued $\mathbf{L F I}$ s, including the well-known da Costa and D'Ottaviano's logic $\mathbf{J 3}$ (and so all of its equivalent presentations, such as LFI1, CLuN or LPT0), as well as Sette's logic $\mathbf{P 1}$, if extended by the inference rules proposed here, will be no longer paraconsistent. Of course, this result is related to the one obtained in Avron and Béziau (2017), which states that for no three-valued paraconsistent logic with implication the replacement property can hold.

The second example deals with the well-known da Costa logic $C_{1}$.

EXAmple 4.2 (da Costa's logic $C_{1}$ )

Newton da Costa introduced in da Costa (1963) his famous hierarchy of paraconsistent systems $C_{n}$ (for $n \geq 1$ ), the first systematic study on paraconsistency in the literature. As discussed above, da Costa's approach was generalized through the notion of $\mathbf{L F I}$ s. The first and stronger system in da Costa's hierarchy is $C_{1}$, which is equivalent (up to language) with Cila. The logic Cila corresponds, with the notation introduced above, to $\mathbf{m b C}\left(\left\{\mathbf{c i}, \mathbf{c l}, \mathbf{c f}, \mathbf{c a}_{\wedge}, \mathbf{c a}_{\vee}, \mathbf{c a}_{\rightarrow}\right\}\right)$. Now, let $\mathbf{R C i l a}$ be the logic $\mathbf{R m b C}\left(\left\{\mathbf{c i}, \mathbf{c l}, \mathbf{c f}, \mathbf{c a}_{\wedge}, \mathbf{c a}_{\vee}, \mathbf{c a}_{\rightarrow}\right\}\right)$; then, this logic derives $(\mathbf{c i w})$. Indeed, as shown in Carnielli and Coniglio (2016a, Proposition 3.1.10), axiom (ciw) is derivable from mbC plus axiom (ci). This being so, by Example 3.7 and the fact that $\perp \stackrel{\text { def }}{=}(\alpha \wedge \neg \alpha) \wedge \neg(\alpha \wedge \neg \alpha)$ is a bottom formula in Cila (hence in RCila) for any $\alpha$ (i.e., $\perp$ implies any other formula), the connective o could be eliminated from the signature of RCila, and so the logic RCila could be defined over the signature $\Sigma_{C}$ (recall Definition 2.2). In that case, RCila would coincide with $\mathbf{R} C_{1}$, the extension of $C_{1}$ by adding the inference rule $\left(\boldsymbol{R}_{\neg}\right)$ (and where the notion of derivation is given as in Definition 2.6). The question is to find a model of RCila (or, equivalently, of $\mathbf{R} C_{1}$ ) which is still paraconsistent.

In Carnielli and Marcos (2002, Theorem 3.51(iv)) it was proved that (IpE) cannot hold in any paraconsistent extension of $\mathbf{C i}$ in which the schema $(\mathbf{d m}): \neg(\alpha \wedge \beta) \rightarrow(\neg \alpha \vee \neg \beta)$ is valid. On the other hand, in Carnielli and Coniglio (2016a, Theorem 3.6.4) it was proved that the logic obtained from $\mathbf{m b C c i w}$ by adding $\left(\mathbf{c a}_{\wedge}\right)$ is equivalent to the logic obtained from $\mathbf{m b C c i w}$ by adding the schema axiom $(\mathbf{d m})$. Since $\mathbf{R C i l a}$ derives $(\mathbf{c i w})$ and $\left(\mathbf{c a}{ }_{\wedge}\right)$, it also derives the schema $(\mathbf{d m})$. Finally, given that RCila is an extension of $\mathbf{C i}$ which satisfies (IpE) and where (dm) is valid, it cannot be paraconsistent, by Carnielli and Marcos 2002, Theorem 3.51(iv)).

The failure of finding a paraconsistent extension of $C_{1}$ enjoying (IpE) was first observed in Urbas (1987, Chapter 5, Theorem 9), by proving that the extension of $C_{n}$ by replacement collapses to classical logic (see also Urbas (1989, Theorem 9) and Sylvan (1990, Section 4)).

\section{Neighborhood semantics for RmbC}

In this section we will introduce a particular case of BALFIs based on powerset Boolean algebras. These structures are in fact equivalent to neighborhood frames for non-normal modal logics, as we shall see in Section 5.1. Moreover, we shall prove in that Section that, with this semantics, $\mathbf{R m b C}$ can be defined within the bimodal version of the minimal modal logic $\mathbf{E}$ (also termed classical modal logic in Chellas (1980, Definition 8.1)). 
Definition 5.1 Let $W$ be a non-empty set. A neighborhood frame for $\mathbf{R m b C}$ over $W$ is a triple $\mathcal{F}=\left\langle W, S_{\neg}, S_{\circ}\right\rangle$ such that $S_{\neg}: \wp(W) \rightarrow \wp(W)$ and $S_{\circ}: \wp(W) \rightarrow \wp(W)$ are functions. $A$ neighborhood model for $\mathbf{R m b C}$ over $\mathcal{F}$ is a pair $\mathcal{M}=\langle\mathcal{F}, d\rangle$ such that $\mathcal{F}$ is a neighborhood frame for $\mathbf{R m b C}$ over $W$ and $d: \mathcal{V} \rightarrow \wp(W)$ is a (valuation) function (given a denumerable set $\mathcal{V}$ of propositional variables).

Definition 5.2 Let $\mathcal{M}=\langle\mathcal{F}, d\rangle$ be a neighborhood model for $\mathbf{R m b C}$ over $\mathcal{F}=\left\langle W, S_{\neg}, S_{\circ}\right\rangle$. It induces a denotation function $\llbracket \cdot \rrbracket_{\mathcal{M}}: \operatorname{For}(\Sigma) \rightarrow \wp(W)$ defined recursively as follows (for simplicity, we will write $\llbracket \varphi \rrbracket$ instead of $\llbracket \varphi \rrbracket_{\mathcal{M}}$ when $\mathcal{M}$ is clear from the context):

$$
\begin{array}{ll}
\llbracket p \rrbracket & =d(p), \text { if } p \in \mathcal{V} ; \\
\llbracket \varphi \wedge \psi \rrbracket & =\llbracket \varphi \rrbracket \cap \llbracket \psi \rrbracket ; \\
\llbracket \varphi \vee \psi \rrbracket & =\llbracket \varphi \rrbracket \cup \llbracket \psi \rrbracket ; \\
\llbracket \varphi \rightarrow \psi \rrbracket & =\llbracket \varphi \rrbracket \rightarrow \llbracket \psi \rrbracket=(W \backslash \llbracket \varphi \rrbracket) \cup \llbracket \psi \rrbracket ; \\
\llbracket \neg \varphi \rrbracket & =(W \backslash \llbracket \varphi \rrbracket) \cup S_{\neg}(\llbracket \varphi \rrbracket) ; \text { and } \\
\llbracket \circ \varphi \rrbracket & =(W \backslash(\llbracket \varphi \rrbracket \cap \llbracket \neg \varphi \rrbracket)) \cap S_{\circ}(\llbracket \varphi \rrbracket)=\left(W \backslash\left(\llbracket \varphi \rrbracket \cap S_{\neg}(\llbracket \varphi \rrbracket)\right)\right) \cap S_{\circ}(\llbracket \varphi \rrbracket) .
\end{array}
$$

Clearly $\llbracket \varphi \rrbracket \cup \llbracket \neg \varphi \rrbracket=W$, but $\llbracket \varphi \rrbracket \cap \llbracket \neg \varphi \rrbracket$ is not necessarily empty. In addition, $\llbracket \varphi \rrbracket \cap \llbracket \neg \varphi \rrbracket \cap$ $\llbracket \circ \varphi \rrbracket=\emptyset$. Intuitively, the functions $S_{\neg}$ and $S_{\circ}$ act as 'regulators' for the values given to the non-standard connectives $\neg$ and $\circ$. Indeed, $S_{\neg}(\llbracket \varphi \rrbracket)$ is the value to be 'added' to the value of the classical negation of $\varphi$ (that is, the Boolean complement of $\llbracket \varphi \rrbracket$ ) in order to allow paraconsistency. In fact, in RmbCciw and its extensions the value of $\circ \varphi$ is the Boolean complement of the value of the contradiction $\varphi \wedge \neg \varphi$. However, in $\mathbf{R m b C}$ the value of $\circ \varphi$ could be less than this, and thus it becomes 'decreased' by intersection with $S_{\circ}(\llbracket \varphi \rrbracket)$.

Definition 5.3 Let $\mathcal{M}=\langle\mathcal{F}, d\rangle$ be a neighborhood model for $\mathbf{R m b C}$.

(1) We say that a formula $\varphi$ is valid (or true) in $\mathcal{M}$, denoted by $\mathcal{M} \Vdash \varphi$, if $\llbracket \varphi \rrbracket=W$.

(2) We say that a formula $\varphi$ is valid w.r.t. neighborhood models, denoted by $\models_{\mathbb{N M}} \varphi$, if $\mathcal{M} \Vdash \varphi$ for every neighborhood model $\mathcal{M}$ for $\mathbf{R m b C}$.

(3) The consequence relation $\models_{\mathbb{N M}}$ induced by neighborhood models for $\mathbf{R m b C}$ is defined as follows: $\Gamma \models_{\mathbb{N M}} \varphi$ if either $\models_{\mathbb{N M}} \varphi$ or there exists a finite, non-empty subset $\left\{\gamma_{1}, \ldots, \gamma_{n}\right\}$ of $\Gamma$ such that $=_{\mathbb{N M}}\left(\gamma_{1} \wedge \ldots \wedge \gamma_{n}\right) \rightarrow \varphi$.

Clearly, $\Gamma \models_{\mathbb{N M}} \varphi$ if either $\llbracket \varphi \rrbracket_{\mathcal{M}}=W$ for every neighborhood model $\mathcal{M}$ for $\mathbf{R m b C}$, or there exists a finite, non-empty subset $\left\{\gamma_{1}, \ldots, \gamma_{n}\right\}$ of $\Gamma$ such that, for every neighborhood model $\mathcal{M}$ for $\mathbf{R m b C}, \bigcap_{i=1}^{n} \llbracket \gamma_{i} \rrbracket_{\mathcal{M}} \subseteq \llbracket \varphi \rrbracket_{\mathcal{M}}$.

Proposition 5.4 Given a neighborhood frame $\mathcal{F}=\left\langle W, S_{\neg}, S_{\circ}\right\rangle$ for $\mathbf{R m b C}$ let $\tilde{ᄀ}$, õ : $\wp(W) \rightarrow$ $\wp(W)$ defined as follows: $\sim(X)=(W \backslash X) \cup S_{\neg}(X)$ and $\tilde{\circ}(X)=\left(W \backslash\left(X \cap S_{\neg}(X)\right) \cap S_{\circ}(X)\right.$. Then $\mathcal{B}_{\mathcal{F}} \stackrel{\text { def }}{=}\langle\wp(W), \cap, \cup, \rightarrow, \tilde{ᄀ}, \tilde{o}, \emptyset, W\rangle$ is a BALFI. Moreover, if $\mathcal{M}=\langle\mathcal{F}, d\rangle$ is a neighborhood model for $\mathbf{R m b C}$ over $\mathcal{F}=\left\langle W, S_{\neg}, S_{\circ}\right\rangle$ then the denotation function $\llbracket \cdot \rrbracket_{\mathcal{M}}$ is a valuation over $\mathcal{B}_{\mathcal{F}}$.

Proof. It is immediate from the definitions.

COROLlaRY 5.5 (Soundness of RmbC w.r.t. neighborhood models) If $\Gamma \vdash_{\mathbf{R m b C}} \varphi$ then $\Gamma \models_{\mathbb{N M}} \varphi$.

Proof. It follows from soundness of RmbC w.r.t. BALFI semantics (Theorem 2.14) and by Proposition 5.4 .

Proposition 5.4 suggests the following : 
Definition 5.6 Let $\mathcal{F}=\left\langle W, S_{\neg}, S_{\circ}\right\rangle$ be a neighborhood frame for $\mathbf{R m b C}$. A formula $\varphi$ is valid in $\mathcal{F}$ if $\mathcal{M} \Vdash \varphi$ for every neighborhood model $\mathcal{M}=\langle\mathcal{F}, d\rangle$ for $\mathbf{R m b C}$ over $\mathcal{F}$.

In order to prove completeness of $\mathbf{R} \mathbf{m b C}$ w.r.t. neighborhood models, Stone's representation theorem for Boolean algebras will be used. This important theorem, first proved in Stone (1936), states that every Boolean algebra is isomorphic to a Boolean subalgebra of $\wp(W)$, for a suitable $W$. This means that, given a Boolean algebra $\mathcal{A}$, there exists a set $W$ and an injective homomorphism $i: \mathcal{A} \rightarrow \wp(W)$ of Boolean algebras. Note that $i(a)=W$ if and only if $a=1$.

TheOREM 5.7 (Completeness of $\mathbf{R m b C}$ w.r.t. neighborhood models) If $\Gamma \models_{\mathbb{N M}} \varphi$ then $\Gamma \vdash_{\mathbf{R m b C}} \varphi$.

Proof. Let $\mathcal{A}_{\text {can }}$ be the Boolean algebra with carrier $A_{\text {can }}=\operatorname{For}(\Sigma) / \equiv$, and let $\mathcal{B}_{\text {can }}$ be the corresponding expansion to $\Sigma_{e}$, as defined in the proof of Theorem 2.15. Let $i: \mathcal{A}_{\text {can }} \rightarrow \wp(W)$ be an injective homomorphism of Boolean algebras, according to Stone's theorem as discussed above. Consider the neighborhood frame $\mathcal{F}_{c a n}=\left\langle W, S_{\neg}, S_{\circ}\right\rangle$ for $\mathbf{R m b C}$ such that the functions $S_{\neg}$ and $S_{\circ}$ satisfy the following: $S_{\neg}(i([\alpha]))=i([\neg \alpha])$, and $S_{\circ}(i([\alpha]))=i([\circ \alpha])$, for every formula $\alpha$ (where $[\alpha]$ denotes the equivalence class of $\alpha$ w.r.t. $\equiv$ ). Observe that these functions are well-defined, since every connective in $\mathbf{R m b C}$ is congruential and $i$ is injective. The values of these functions outside the image of $i$ can be arbitrary. Now, let $\mathcal{M}_{\text {can }}=\left\langle\mathcal{F}_{\text {can }}, d_{\text {can }}\right\rangle$ be the neighborhood model for $\mathbf{R m b C}$ such that $d_{c a n}(p) \stackrel{\text { def }}{=} i([p])$, for every propositional variable $p$.

Fact: $\llbracket \alpha \rrbracket_{\mathcal{M}_{\text {can }}}=i([\alpha])$, for every formula $\alpha$.

The proof of the Fact will be done by induction on the complexity of the formula $\alpha$ (recalling Definition 5.2 for $\left.\llbracket \cdot \rrbracket_{\mathcal{M}}\right)$. The complexity of $\circ \alpha$ is taken to be stricty greater than the complexity of $\neg \alpha$. The case for $\alpha$ atomic or $\alpha=\beta \# \gamma$ for $\# \in\{\wedge, \vee, \rightarrow\}$ is clear, by the very definitions and by induction hypothesis. Now, suppose that $\alpha=\neg \beta$. By induction hypothesis, $\llbracket \beta \rrbracket=i([\beta])$. Observe that $\sim[\beta] \leq[\neg \beta]$ in $\mathcal{A}_{\text {can }}$ (where $\sim$ denotes the Boolean complement in $\mathcal{A}_{\text {can }}$ ), hence $W \backslash i([\beta])=i(\sim[\beta]) \subseteq i([\neg \beta])$. Thus,

$$
\begin{aligned}
\llbracket \neg \beta \rrbracket & =(W \backslash \llbracket \beta \rrbracket) \cup S_{\neg}(\llbracket \beta \rrbracket)=(W \backslash i([\beta])) \cup S_{\neg}(i([\beta])) \\
& =(W \backslash i([\beta])) \cup i([\neg \beta])=i([\neg \beta]) .
\end{aligned}
$$

Finally, let $\alpha=\circ \beta$. Since $[\circ \beta] \leq \sim([\beta] \wedge[\neg \beta])$ in $\mathcal{A}_{\text {can }}$ then $i([\circ \beta]) \subseteq W \backslash(i([\beta]) \cap i([\neg \beta]))$. Hence, by induction hypothesis,

$$
\begin{aligned}
\llbracket \circ \beta \rrbracket & =(W \backslash(i([\beta]) \cap i([\neg \beta]))) \cap S_{\circ}(i([\beta]) \\
& =(W \backslash(i([\beta]) \cap i([\neg \beta]))) \cap i([\circ \beta])=i([\circ \beta]) .
\end{aligned}
$$

This concludes the proof of the Fact.

Because of the Fact, $\mathcal{M}_{\text {can }} \Vdash \alpha$ iff $i([\alpha])=W$ iff $[\alpha]=1$ iff $\vdash_{\mathbf{R m b C}} \alpha$. Now, suppose that $\Gamma \models_{\mathbb{N M}} \varphi$. If $\models_{\mathbb{N M}} \varphi$ then, in particular, $\mathcal{M}_{\text {can }} \Vdash \varphi$ and so $\vdash_{\mathbf{R m b C}} \varphi$. From this, $\Gamma \vdash_{\mathbf{R m b C}} \varphi$. On the other hand, suppose that there exists a finite, non-empty subset $\left\{\gamma_{1}, \ldots, \gamma_{n}\right\}$ of $\Gamma$ such that $\models_{\mathbb{N M}}\left(\gamma_{1} \wedge \ldots \wedge \gamma_{n}\right) \rightarrow \varphi$. By reasoning as above, it follows that $\vdash_{\mathbf{R m b C}}\left(\gamma_{1} \wedge \ldots \wedge \gamma_{n}\right) \rightarrow \varphi$ and so $\Gamma \vdash_{\mathbf{R m b C}} \varphi$ as well.

\subsection{RmbC is definable within the minimal bimodal modal logic}

This section shows that $\mathbf{R m b C}$ is definable within the bimodal version of the minimal modal logic E, also called classical modal logic in Chellas (1980, Definition 8.1). In terms of combination 
of modal logics, this bimodal logic is equivalent to the fusion (or, equivalently, the constrained fibring by sharing the classical connectives) of $\mathbf{E}$ with itself 12 This means that the minimal non-normal modal logic with two independent modalities $\square_{1}$ and $\square_{2}$, which will be denoted by $\mathbf{E} \oplus \mathbf{E}$, contains RmbC, the minimal self-extensional LFI. As we shall see, in the case of $\mathbf{R m b C}$ both modalities are required for defining the two non-classical conectives $\neg$ and $\circ$. For RmbCciw and its extensions only one modality is needed, since $\circ$ can be taken as a derived operator (recall Example 3.7). Hence RmbCciw is definable in $\mathbf{E}$.

The minimal (or classical) modal logic $\mathbf{E}$ was introduced by Montague (1970) and Scott (1970) with semantics given by neighborhood models, which generalize the usual Kripke models for modal logic. Neighborhood semantics has become one of the main mechanisms for providing semantics for non-normal modal logics as well as for some normal modal logics that are not complete w.r.t. Kripke models (see e.g. Gabbay, 1975). Among the first important applications of neighborhood semantics we count the well-known book on counterfactual conditionals by Lewis (1973). Ever since, it has found many successful applications in areas of conditional, deontic, and epistemic logic (see, e.g., Pacuit, 2017). For convenience, the definition of modal $\operatorname{logic} \mathbf{E}$ will be briefly surveyed below.

Definition 5.8 (Chellas (1980), Definition 7.1) $A$ minimal model is a triple $\mathcal{N}=\langle W, N, d\rangle$ such that $W$ is a non-empty set and $N: W \rightarrow \wp(\wp(W))$ and $d: \mathcal{V} \rightarrow \wp(W)$ are functions. The class of minimal models will be denoted by $\mathcal{C}_{\mathcal{M}}$.

Let $\Sigma_{m}=\{\wedge, \vee, \rightarrow, \sim, \square, \diamond\}$ and $\Sigma_{b m}=\left\{\wedge, \vee, \rightarrow, \sim, \square_{1}, \diamond_{1}, \square_{2}, \diamond_{2}\right\}$ be the signatures introduced in Definition 2.2. The class of models $\mathcal{C}_{\mathcal{M}}$ induces a modal consequence relation defined as follows:

Definition 5.9 (Chellas (1980), Definition 7.2) Let $\mathcal{N}$ be a minimal model and $w \in W . \mathcal{N}$ is said to satisfy a formula $\varphi \in \operatorname{For}\left(\Sigma_{m}\right)$ in $w$, denoted by $\models_{w}^{\mathcal{N}} \varphi$, according to the following recursive definition (here $\llbracket \varphi \rrbracket^{\mathcal{N}}$ stands for the set $\left\{w \in W: \models_{w}^{\mathcal{N}} \varphi\right\}$, the denotation of $\varphi$ in $\mathcal{N}$ ):

1. if $p$ is a propositional variable then $\models_{w}^{\mathcal{N}} p \quad$ iff $w \in d(p)$;

2. $\quad=_{w}^{\mathcal{N}} \sim \alpha$ iff $\quad \nvdash_{w}^{\mathcal{N}} \alpha$;

3. $\models{ }_{w}^{\mathcal{N}} \alpha \wedge \beta \quad$ iff $\quad \models_{w}^{\mathcal{N}} \alpha$ and $\models_{w}^{\mathcal{N}} \beta$;

4. $\models_{w}^{\mathcal{N}} \alpha \vee \beta$ iff $\models_{w}^{\mathcal{N}} \alpha$ or $\models_{w}^{\mathcal{N}} \beta$;

5. $\quad \models_{w}^{\mathcal{N}} \alpha \rightarrow \beta \quad$ iff $\quad \nvdash_{w}^{\mathcal{N}} \alpha$ or $\models_{w}^{\mathcal{N}} \beta$;

6. $\quad \models{ }_{w}^{\mathcal{N}} \square \alpha$ iff $\llbracket \alpha \rrbracket^{\mathcal{N}} \in N(w)$;

7. $\quad=_{w}^{\mathcal{N}} \diamond \alpha \quad$ iff $\left(W \backslash \llbracket \alpha \rrbracket^{\mathcal{N}}\right) \notin N(w)$.

A formula $\varphi$ is true in $\mathcal{N}$ if $\llbracket \varphi \rrbracket^{\mathcal{N}}=W$, and it is valid w.r.t. $\mathcal{C}_{\mathcal{M}}$, denoted by $\models^{\mathcal{C}_{\mathcal{M}}} \varphi$, if it is true in every minimal model. The degree-preserving consequence w.r.t. $\mathcal{C}_{\mathcal{M}}$ can be defined analogously to the one for neighborhood semantics for RmbC given in Definition 5.3. Namely, $\Gamma \models^{\mathcal{C}_{\mathcal{M}}} \varphi$ if either $\models^{\mathcal{C}_{\mathcal{M}}} \varphi$, or there exists a finite, non-empty subset $\left\{\gamma_{1}, \ldots, \gamma_{n}\right\}$ of $\Gamma$ such that $\models^{\mathcal{C}_{\mathcal{M}}}\left(\gamma_{1} \wedge \ldots \wedge \gamma_{n}\right) \rightarrow \varphi$. The latter is equivalent to $\bigcap_{i=1}^{n} \llbracket \gamma_{i} \rrbracket^{\mathcal{N}} \subseteq \llbracket \varphi \rrbracket^{\mathcal{N}}$.

Definition 5.10 (Chellas (1980), Definition 8.1) The minimal modal logic (or classical modal logic) $\mathbf{E}$ is defined by means of a Hilbert calculus over the signature $\Sigma_{m}$ obtained by adding to the Hilbert calculus for $\mathbf{C P L}^{+}$(recall Definition 2.3) the following axiom schemas and rules:

\footnotetext{
${ }^{12}$ For the basic notions of combining logics the reader can consult Carnielli and Coniglio (2020) and Carnielli et al. (2008).
} 


$$
\begin{gathered}
\alpha \vee \sim \alpha \\
\alpha \rightarrow(\sim \alpha \rightarrow \beta) \\
\diamond \alpha \leftrightarrow \sim \square \sim \alpha \\
\frac{\alpha \leftrightarrow \beta}{\square \alpha \leftrightarrow \square \beta}
\end{gathered}
$$

The notion of derivation in $\mathbf{E}$ is defined as for $\mathbf{R m b C}$, recall Definition 2.6. Note that $(\mathbf{P E M})$ and $(\mathbf{e x p})$, together with $\mathbf{C P L}{ }^{+}$, guarantee that $\mathbf{E}$ is an expansion of propositional classical logic by adding the modalities $\square$ and $\diamond$ which are interdefinable as usual, and such that both are congruential. That is, $\mathbf{E}$ satisfies replacement.

Theorem 5.11 (Chellas (1980), Section 9.2) The logic $\mathbf{E}$ is sound and complete w.r.t. the semantics in $\mathcal{C}_{\mathcal{M}}$, namely: $\Gamma \vdash_{\mathbf{E}} \varphi$ iff $\Gamma \models^{\mathcal{C}_{\mathcal{M}}} \varphi$.

Definition 5.12 (Minimal bimodal logic) The minimal bimodal logic $\mathbf{E} \oplus \mathbf{E}$ is defined by means of a Hilbert calculus over signature $\Sigma_{b m}$ obtained by adding to the Hilbert calculus for $\mathbf{C P L}^{+}$ the following axiom schemas and rules, for $i=1,2$ :

$$
\begin{gathered}
\alpha \vee \sim \alpha \\
\alpha \rightarrow(\sim \alpha \rightarrow \beta) \\
\diamond_{i} \alpha \leftrightarrow \sim \square_{i} \sim \alpha \\
\frac{\alpha \leftrightarrow \beta}{\square_{i} \alpha \leftrightarrow \square_{i} \beta}
\end{gathered}
$$

Observe that $\mathbf{E} \oplus \mathbf{E}$ is obtained from $\mathbf{E}$ by 'duplicating' the modalities. There is no relationship between $\square_{1}$ and $\square_{2}$ and so $\diamond_{1}$ and $\diamond_{2}$ are also independent.

The semantics of $\mathbf{E} \oplus \mathbf{E}$ is given by the class $\mathcal{C}_{\mathcal{M}}^{\prime}$ of structures of the form $\mathcal{N}=\left\langle W, N_{1}, N_{2}, d\right\rangle$ such that $W$ is a non-empty set and $N_{i}: W \rightarrow \wp(\wp(W)$ ) (for $i=1,2)$ and $d: \mathcal{V} \rightarrow \wp(W)$ are functions. The denotation $\llbracket \varphi \rrbracket^{\mathcal{N}}$ of a formula $\varphi \in \operatorname{For}\left(\Sigma_{b m}\right)$ in $\mathcal{N}$ is defined by an obvious adaptation of Definition 5.9 to $\operatorname{For}\left(\Sigma_{b m}\right)$. By defining the consequence relations $\vdash_{\mathbf{E} \oplus \mathbf{E}}$ and $\models \mathcal{C}_{\mathcal{M}}^{\prime}$ in analogy to the ones for $\mathbf{E}$, it is straightforward to adapt the proof of soundness and completeness of $\mathbf{E}$ to the bimodal case.

Theorem 5.13 The logic $\mathbf{E} \oplus \mathbf{E}$ is sound and complete w.r.t. the semantics in $\mathcal{C}_{\mathcal{M}}^{\prime}$, namely: $\Gamma \vdash_{\mathbf{E} \oplus \mathbf{E}} \varphi$ iff $\Gamma \models^{\mathcal{C}_{\mathcal{M}}^{\prime}} \varphi$.

From the point of view of combining logics, $\mathbf{E} \oplus \mathbf{E}$ is the fusion (or, equivalently, the constrained fibring by sharing the classical connectives) of $\mathbf{E}$ with itself (see Carnielli and Coniglio, 2020 Carnielli et al. 2008).

Finally, it will be shown that $\mathbf{R m b C}$ can be defined inside $\mathbf{E} \oplus \mathbf{E}$ by means of the following abbreviations, which are motivated by Definition 5.2 (having in mind that $\square_{1}$ and $\square_{2}$ will be interpreted by the mappings $S_{\neg}$ and $S_{\circ}$, respectively):

$$
\neg \varphi \stackrel{\text { def }}{=} \varphi \rightarrow \square_{1} \varphi \text { and } \circ \varphi \stackrel{\text { def }}{=} \sim\left(\varphi \wedge \square_{1} \varphi\right) \wedge \square_{2} \varphi .
$$

In order to see this, observe that any function $N: W \rightarrow \wp(\wp(W))$ induces a unique function $S: \wp(W) \rightarrow \wp(W)$ given by $S(X)=\{w \in W: X \in N(w)\}$. Conversely, any function $S$ : 
$\wp(W) \rightarrow \wp(W)$ induces a function $N: W \rightarrow \wp(\wp(W))$ given by $N(w)=\{X \subseteq W: w \in S(X)\}$. Both assignments $N \mapsto S$ and $S \mapsto N$ are inverses of each other. From this, a structure (or minimal model) $\mathcal{N}=\left\langle W, N_{1}, N_{2}, d\right\rangle$ for $\mathbf{E} \oplus \mathbf{E}$ can be transformed into a neighborhood model $\mathcal{M}=\left\langle W, S_{\neg}, S_{\circ}, d\right\rangle$ for $\mathbf{R m b C}$ such that $S_{\neg}$ and $S_{\circ}$ are obtained, respectively, from the functions $N_{1}$ and $N_{2}$ as indicated above. Observe that

$$
w \in \llbracket \square_{1} \varphi \rrbracket^{\mathcal{N}} \text { iff } \quad \models_{w}^{\mathcal{N}} \square_{1} \varphi \text { iff } \llbracket \varphi \rrbracket^{\mathcal{N}} \in N_{1}(w) \text { iff } w \in S_{\neg}\left(\llbracket \varphi \rrbracket^{\mathcal{N}}\right) .
$$

That is, $S_{\neg}\left(\llbracket \varphi \rrbracket^{\mathcal{N}}\right)=\llbracket \square_{1} \varphi \rrbracket^{\mathcal{N}}$. Analogously, $S_{\circ}\left(\llbracket \varphi \rrbracket^{\mathcal{N}}\right)=\llbracket \square_{2} \varphi \rrbracket^{\mathcal{N}}$. From this, it is easy to prove by induction on the complexity of the formula $\varphi \in \operatorname{For}(\Sigma)$ that $\llbracket \varphi \rrbracket_{\mathcal{M}}=\llbracket \varphi^{t} \rrbracket^{\mathcal{N}}$, where $\varphi^{t}$ is the formula over the signature $\Sigma_{b m}$ obtained from $\varphi$ by replacing any ocurrence of the connectives $\neg$ and $\circ$ by the corresponding abbreviations, as indicated above. Conversely, any neighborhood model $\mathcal{M}=\left\langle W, S_{\neg}, S_{\circ}, d\right\rangle$ for $\mathbf{R m b C}$ gives origin to a unique minimal model $\mathcal{N}=\left\langle W, N_{1}, N_{2}, d\right\rangle$ for $\mathbf{E} \oplus \mathbf{E}$ such that $\llbracket \varphi \rrbracket_{\mathcal{M}}=\llbracket \varphi^{t} \rrbracket^{\mathcal{N}}$ for every formula $\varphi \in F o r(\Sigma)$. That is, the class of minimal models for $\mathbf{E} \oplus \mathbf{E}$ coincides (up to presentation) with the class of neighborhood models for $\mathbf{R m b C}$, and both classes validate the same formulas over the signature $\Sigma$ of $\mathbf{R m b C}$. From this, Corollary 5.5. Theorem 5.7 and Theorem 5.13 we show that $\mathbf{R m b C}$ is definable within $\mathbf{E} \oplus \mathbf{E}$ :

Theorem 5.14 The logic $\mathbf{R m b C}$ is definable within $\mathbf{E} \oplus \mathbf{E}$, in the following sense: $\Gamma \vdash_{\mathbf{R m b C}} \varphi$ iff $\Gamma^{t} \vdash_{\mathbf{E} \oplus \mathbf{E}} \varphi^{t}$ for every $\Gamma \cup\{\varphi\} \subseteq \operatorname{For}(\Sigma)$, where $\Gamma^{t}=\left\{\psi^{t}: \psi \in \Gamma\right\}$.

The main result obtained in this section, namely Theorem 5.14, establishes an interesting relation between non-normal modal logics and paraconsistent logics.

Connections between modalities and paraconsistency, or proposals emphasizing a connection between negations, denial, and impossibility, are well-known in the literature. Segerberg (1982, p. 128) suggests the possibility of studying the (unexplored at that time) modal notion of ' $\varphi$ is non-necessary', namely $\sim \square \varphi$. Along the same lines, Došen (1984) investigates modal operators corresponding to impossibility and non-necessity in systems analogous to the modal system $\mathbf{K}$ based on intuitionistic (instead of classical) logic. A system where impossibility is equivalent to intuitionistic negation is also investigated (proving soundness and completeness). Došen (1986) investigates negation as a modal impossibility operator added to a negationless logic. In turn, Vakarelov (1989 ${ }^{13}$ builds upon a distributive (lattice) logic to introduce a class of negations (generalizing intuitionistic negation) termed regular negations by drawing upon the notion of theory consistency/compatibility. Some convenient semantical simplifications giving rise to the special subclasses of normal and standard negations are also discussed. In an analogous manner, by drawing upon the dual notion of theory completeness, Vakarelov introduces the dual class of co-regular (as well as co-normal and co-standard) negations. Vakarelov also discusses paraconsistent and paracomplete negations and relates them to particular subclasses of regular and co-regular logics. Along similar lines, Dunn and Zhou (2005) (drawing upon Dunn (1993)) study two families of negative modal operators interpreted as impossibility and (dually) as non-necessity, each represented in an ordered structure termed "(dual) kite of negations".

More recently, Béziau (2002, 2005) proposes to examine a paraconsistent negation defined in the modal system S5 as $\neg \varphi \stackrel{\text { def }}{=} \nabla \sim \varphi$ (which is equivalent to $\sim \square \varphi$ ). Historically situated between the early works by Došen and Vakarelov mentioned above and Béziau's proposal, a similar way of defining a paraconsistent negation inside a modal logic has been regarded in de Araújo et al. (1987), where the authors propose a Kripke-style semantics for Sette's three-valued paraconsistent logic P1 based on Kripke frames for the modal logic $\mathbf{T}$. This result was improved in Carnielli and Lima-Marques (1999), by showing that $\mathbf{P 1}$ can be interpreted in $\mathbf{T}$ by means of Kripke frames having at most two worlds.

\footnotetext{
${ }^{13}$ This is a summary of Vakarelov's PhD thesis "Theory of negation in certain logical systems. Algebraic and semantical approach", University of Warsaw, 1976.
} 
Several authors have explored the possibility of defining such paraconsistent negation in other modal logics such as B (Avron and Zamansky, 2016), S4 (Coniglio and Prieto-Sanabria, 2017) and even weaker modal systems (Bueno-Soler and Carnielli, 2017). In such context, Marcos (2005b) proposes, besides the paraconsistent negation defined as above, the definition of a consistency connective within a modal system by means of the formula $\circ \varphi \stackrel{\text { def }}{=} \varphi \rightarrow \square \varphi$. In that paper it is shown that any normal modal logic in which the schema $\varphi \rightarrow \square \varphi$ is not valid gives origin to an LFI in this way. Moreover, it is shown that it is also possible to start from a "modal LFI", over the signature $\Sigma$ of LFIs, in which the paraconsistent negation and the consistency connective feature a Kripke-style semantics, defining the modal necessity operator by means of the formula $\square \varphi \stackrel{\text { def }}{=} \sim \neg \varphi$ (where $\sim$ is the strong negation defined in terms of $\neg$ and $\circ$ as in mbC, recall Remark $3.2(2)$ ). This shows that 'reasonable' normal modal logics and LFIs with Kripke-style semantics are two faces of the same coin.

Our Theorem 5.14 partially extends this relationship to the realm of non-normal modal logics. The result we have obtained is partial, in the sense that the minimum bimodal nonnormal modal logics gives origin to $\mathbf{R} \mathbf{m b C}$, but the converse does not seem to be true. Namely, starting from $\mathbf{R m b C}$ it is not obvious that the modalities $\square_{1}$ and $\square_{2}$ could be defined by means of formulas in the signature $\Sigma$. This topic deserves further investigation.

\subsection{Neighborhood models for axiomatic extensions of RmbC}

In this section $A x$ will denote a set formed by one or more of the axiom schemas introduced in Definition 3.1 with the exception of $\left(\mathbf{c a}_{\#}\right)$ for $\# \in\{\wedge, \vee, \rightarrow\}$ (given the limitations to paraconsistency imposed to logic RCila (recall Example 4.2). Let $\mathbb{N M}(A x)$ the class of neighborhood frames in which every schema in $A x$ is valid. Define the consequence relation $\models_{\mathbb{N M}(A x)}$ in the obvious way. By adapting the previous results it is easy to prove the following:

Theorem 5.15 (Soundness and completeness of $\mathbf{R m b C}(A x)$ w.r.t. $\mathbb{N M}(A x)$ ) Let $\Gamma \cup\{\varphi\} \subseteq$ For $(\Sigma)$. Then: $\Gamma \vdash_{\mathbf{R m b C}(A x)} \varphi$ if and only if $\Gamma \models_{\mathbb{N M}(A x)} \varphi$.

The class of neighborhood frames that validates each of the axioms of $A x$ can be easily characterized:

Proposition 5.16 Let $\mathcal{F}$ be a neighborhood frame for $\mathbf{R m b C}$. Then:

(1) (ciw) is valid in $\mathcal{F}$ iff $W \backslash\left(X \cap S_{\neg}(X)\right) \subseteq S_{\circ}(X)$, for every $X \subseteq W$;

(2) (ci) is valid in $\mathcal{F}$ iff $W \backslash\left(X \cap S_{\neg}(X)\right) \subseteq S_{\circ}(X) \backslash S_{\neg}\left(\left(W \backslash\left(X \cap S_{\neg}(X)\right)\right) \cap S_{\circ}(X)\right)$, for every $X \subseteq W$;

(3) (cl) is valid in $\mathcal{F}$ iff $S_{\neg}\left(X \cap S_{\neg}(X)\right) \subseteq W \backslash\left(X \cap S_{\neg}(X)\right) \subseteq S_{\circ}(X)$, for every $X \subseteq W$;

(4) (cf) is valid in $\mathcal{F}$ iff $\left(X \backslash S_{\neg}(X)\right) \cup S_{\neg}\left(X \rightarrow S_{\neg}(X)\right) \subseteq X$, for every $X \subseteq W$;

(5) (ce) is valid in $\mathcal{F}$ iff $X \subseteq\left(X \backslash S_{\neg}(X)\right) \cup S_{\neg}\left(X \rightarrow S_{\neg}(X)\right)$, for every $X \subseteq W$.

Recall the minimal bimodal logic $\mathbf{E} \oplus \mathbf{E}$ studied in Section 5.1. If $\varphi$ is a formula in $\operatorname{For}\left(\Sigma_{b m}\right)$ then $\mathbf{E} \oplus \mathbf{E}(\varphi)$ will denote the extension of $\mathbf{E} \oplus \mathbf{E}$ by adding $\varphi$ as an axiom schema. Let $\mathcal{C}_{\mathcal{M}}^{\prime}(\varphi)$ be the class of structures (i.e., minimal models) $\mathcal{N}$ for $\mathbf{E} \oplus \mathbf{E}$ such that $\varphi$ is valid in $\mathcal{N}$ (as an axiom schema). Theorem 5.13 can be extended to prove that the $\operatorname{logic} \mathbf{E} \oplus \mathbf{E}(\varphi)$ is sound and complete w.r.t. the semantics in $\mathcal{C}_{\mathcal{M}}^{\prime}(\varphi)$. From this, and taking into account the representability of $\mathbf{R m b C}$ within $\mathbf{E} \oplus \mathbf{E}$ (Theorem 5.14) and the equivalence between minimal models for $\mathbf{E} \oplus \mathbf{E}$ and neighborhood models for RmbC discussed right before Theorem 5.14, Proposition 5.16 can be recast as follows:

Corollary 5.17

(1) $\mathbf{R m b C}($ ciw $)$ is definable in $\mathbf{E} \oplus \mathbf{E}\left(\sim\left(\varphi \wedge \square_{1} \varphi\right) \rightarrow \square_{2} \varphi\right)$; 
(2) $\mathbf{R m b C}(\mathbf{c i})$ is definable in $\mathbf{E} \oplus \mathbf{E}\left(\sim\left(\varphi \wedge \square_{1} \varphi\right) \rightarrow\left(\square_{2} \varphi \wedge \sim \square_{1}\left(\sim\left(\varphi \wedge \square_{1} \varphi\right) \wedge \square_{2} \varphi\right)\right)\right)$ or, equivalenty, in $\mathbf{E} \oplus \mathbf{E}\left(\left(\square_{2} \varphi \rightarrow \square_{1}\left(\sim\left(\varphi \wedge \square_{1} \varphi\right) \wedge \square_{2} \varphi\right)\right) \rightarrow\left(\varphi \wedge \square_{1} \varphi\right)\right)$;

(3) $\mathbf{R m b C}(\mathbf{c l})$ is definable in $\mathbf{E} \oplus \mathbf{E}\left(\left(\square_{1}\left(\varphi \wedge \square_{1} \varphi\right) \rightarrow \sim\left(\varphi \wedge \square_{1} \varphi\right)\right) \wedge\left(\sim\left(\varphi \wedge \square_{1} \varphi\right) \rightarrow \square_{2} \varphi\right)\right)$;

(4) $\mathbf{R m b C}(\mathbf{c f})$ is definable in $\mathbf{E} \oplus \mathbf{E}\left(\left(\left(\varphi \wedge \sim \square_{1} \varphi\right) \vee \square_{1}\left(\varphi \rightarrow \square_{1} \varphi\right)\right) \rightarrow \varphi\right)$;

(5) $\mathbf{R m b C}(\mathbf{c e})$ is definable in $\mathbf{E} \oplus \mathbf{E}\left(\varphi \rightarrow\left(\left(\varphi \wedge \sim \square_{1} \varphi\right) \vee \square_{1}\left(\varphi \rightarrow \square_{1} \varphi\right)\right)\right)$.

We have, in fact, that $\mathbf{R m b C}(\mathbf{c i w})$ and its extension $\mathbf{R m b C}(\mathbf{c i})$ are definable in $\mathbf{E}$. Recall from Example 3.7 that for them $\circ$ can be defined as a derived operator.

\section{Truth-preserving (or global) semantics}

As mentioned in Section 2, the BALFI semantics for $\mathbf{R m b C}$, as well as its neighborhood semantics presented in Section 5 , is degree-preserving instead of truth-preserving (using the terminology from Bou et al. (2009)). This requires adapting, in a coherent way, the usual definition of derivation from premises in a Hilbert calculus (recall Definition 2.6). This is in fact the methodology adopted with most normal modal logics in which the semantics is local, thus recovering the deduction metatheorem. But it is also possible to consider global (or truth-preserving) semantics, as it is usually done with algebraic semantics. This leads us to propose the logic $\mathbf{R m b C}$, which is defined by the same Hilbert calculus than the one for $\mathbf{R m b C}$, but now derivations from premises in $\mathbf{R m b C} \mathbf{C}^{*}$ are defined as usual in Hilbert calculi.

Definition 6.1 The logic $\mathbf{R} \mathbf{m b C} \mathbf{C}^{*}$ is defined by the same Hilbert calculus over signature $\Sigma$ than $\mathbf{R m b C}$, that is, by adding to $\mathbf{m b C}$ the inference rules $\left(\boldsymbol{R}_{\neg}\right)$ and $\left(\boldsymbol{R}_{\circ}\right)$.

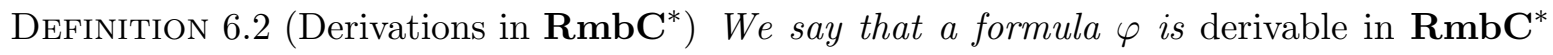
from $\Gamma$, and we write $\Gamma \vdash_{\mathbf{R m b C}^{*}} \varphi$, if there exists a finite sequence of formulas $\varphi_{1} \ldots \varphi_{n}$ such that $\varphi_{n}$ is $\varphi$ and, for every $1 \leq i \leq n$, either $\varphi_{i}$ is an instance of an axiom schema of $\mathbf{R m b C}$, or $\varphi_{i} \in \Gamma$, or $\varphi_{i}$ is the consequence of some inference rule of $\mathbf{R m b C}$ whose premises appear in the sequence $\varphi_{1} \ldots \varphi_{i-1}$.

Now, the degree-preserving BALFI semantics for $\mathbf{R m b C}$ given in Definition 2.12 is replaced by a truth-preserving consequence relation for $\mathbf{R} \mathbf{m b C} \mathbf{C}^{*}$. Notice however that, by virtue of the self-extensionality, this semantical notion will not require finitariness by definition: indeed, after proving soundness and completeness, finitariness of the semantical consequence relation (that is, the semantical compactness theorem for $\mathbf{R} \mathbf{m b} \mathbf{C}^{*}$ ) will be obtained as a corollary.

DeFinition 6.3 (Truth-preserving BALFI semantics)

Let $\Gamma \cup\{\varphi\}$ be a set of formulas in For $(\Sigma)$. We say that $\varphi$ is a truth-preserving (or global) consequence of $\Gamma$ in the variety $\mathbb{B I}$ of BALFIs, denoted by $\Gamma \models_{\mathbb{B I}}^{g} \varphi$, if, for every BALFI $\mathcal{B}$ and every valuation $v$ over it, $v(\varphi)=1$ whenever $v(\gamma)=1$ for every $\gamma \in \Gamma$.

TheOREM 6.4 (Soundness and completeness of $\mathbf{R m b C ^ { * }}$ w.r.t. truth-preserving semantics) For every $\Gamma \cup\{\varphi\} \subseteq$ For $(\Sigma): \Gamma \vdash_{\mathbf{R m b C}^{*}} \varphi$ iff $\Gamma \models_{\mathbb{B I}}^{g} \varphi$.

Proof. (Soundness) The proof is a trivial adaptation of the argument given for $\mathbf{R m b C}$ in the proof of Theorem 2.14 .

(Completeness) It can be obtained by a slight adaptation of the proof of Theorem 2.15 given above. Thus, suppose that $\Gamma \nvdash_{\mathbf{R m b C}^{*}} \varphi$. Define the following relation on $\operatorname{For}(\Sigma): \alpha \equiv_{\Gamma} \beta$ iff $\Gamma \vdash{ }_{\mathbf{R m b C}^{*}} \alpha \leftrightarrow \beta$. By similar arguments to the ones given in the proof of Theorem 2.15, it is easy to see that $\equiv_{\Gamma}$ is an equivalence relation. Moreover, it is a congruence on $\operatorname{For}(\Sigma)$ : indeed, the only connectives that deserve special attention are $\neg$ and $\circ$. However, since rules $\left(\mathbf{R}_{\neg}\right)$ and $\left(\mathbf{R}_{\circ}\right)$ can be used without restrictions in derivations in $\mathbf{R} \mathbf{m b C} \mathbf{C}^{*}$, it follows that, for $\# \in\{\neg, \circ\}$, 
$\# \alpha \equiv_{\Gamma} \# \beta$ provided that $\alpha \equiv_{\Gamma} \beta$. This being so, the quotient set $A_{\text {can }}^{\Gamma} \stackrel{\text { def }}{=} \operatorname{For}(\Sigma) / \equiv_{\Gamma}$ is the carrier of a BALFI, denoted by $\mathcal{B}_{\text {can }}^{\Gamma}$, with operations given as follows (where $[\alpha]_{\Gamma}$ denotes the equivalence class of $\alpha$ w.r.t. $\left.\equiv_{\Gamma}\right):[\alpha]_{\Gamma} \#[\beta]_{\Gamma} \stackrel{\text { def }}{=}[\alpha \# \beta]_{\Gamma}$, for $\# \in\{\wedge, \vee, \rightarrow\} ; \#[\alpha]_{\Gamma} \stackrel{\text { def }}{=}[\# \alpha]_{\Gamma}$, for $\# \in\{\neg, \circ\} ; 0 \stackrel{\text { def }}{=}[\alpha \wedge \neg \alpha \wedge \circ \alpha]_{\Gamma}$ and $1 \stackrel{\text { def }}{=}[\alpha \vee \neg \alpha]_{\Gamma}$ (for any $\alpha$ ). Now, let $v_{\text {can }}^{\Gamma}: \operatorname{For}(\Sigma) \rightarrow$ $A_{c a n}^{\Gamma}$ given by $v_{c a n}^{\Gamma}(\alpha)=[\alpha]_{\Gamma}$. Clearly $v_{\text {can }}^{\Gamma}$ is a valuation over $\mathcal{B}_{c a n}^{\Gamma}$ such that $v_{\text {can }}^{\Gamma}(\alpha)=1$ iff $\Gamma \vdash_{\mathbf{R m b C}^{*}} \alpha$. From this, $v_{\text {can }}^{\Gamma}(\alpha)=1$ for every $\alpha \in \Gamma$, while $v_{\text {can }}^{\Gamma}(\varphi) \neq 1$. This shows that $\Gamma \not \nvdash_{\mathbb{B I}}^{g} \varphi$.

Corollary 6.5 (Semantical compactness of $\mathbf{R m b C}^{*}$ ) If $\Gamma \models_{\mathbb{B I}}^{g} \varphi$ then there exists a finite subset $\Gamma_{0}$ of $\Gamma$ such that $\Gamma_{0} \models_{\mathbb{B I}}^{g} \varphi$.

Proof. It is an immediate consequence of Theorem 6.4 and the fact that the consequence relation $\vdash_{\mathbf{R m b C}^{*}}$ is finitary, by definition.

REMARK 6.6 The definition of truth-preserving semantics restricts the number of paraconsistent models for $\mathbf{R m b C} \mathbf{C}^{*}$. Indeed, let $p$ and $q$ be two different propositional variables. In order to show that $p, \neg p \forall_{\mathbb{B I}}^{g} q$, there must be a BALFI $\mathcal{B}$ and a valuation $v$ over $\mathcal{B}$ such that $v(p)=v(\neg p)=1$ but $v(q) \neq 1$. That is, $\mathcal{B}$ must be such that $\neg 1=1$. Since $\neg 0=1$, it follows that $\neg \neg 0=\neg 1=1 \not \leq 0$ in $\mathcal{B}$. This shows that there is no paraconsistent extension of $\mathbf{R m b \mathbf { C } ^ { * }}$ which satisfies axiom (cf). In particular, there is no paraconsistent extension of $\mathbf{R m b \mathbf { C } ^ { * }}$ satisfying axioms (cf) and (ci). Thus, the open problems solved in Examples 3.9 and 3.10 have a negative answer in this setting. This shows that the truth-preserving approach is much more restricted than the degree-preserving approach in terms of paraconsistency.

In any case, there are still paraconsistent BALFIs for the truth-preserving logic $\mathbf{R m b C} \mathbf{C}^{*}$ (namely, the ones such that $\neg 1=1$ ). The situation is quite different in the realm of fuzzy logics: in Coniglio et al. (2014) and Ertola et al. (2015), among others, the degree-preserving companion of several fuzzy logics has been studied, showing that their usual truth-preserving consequence relations are never paraconsistent.

The distinction between local and global reasoning has been studied by A. Sernadas and his collaborators (for a brief exposition see, for instance, Carnielli et al. (2008), Section 2.3 in Chapter 2). From the proof-theoretical perspective, the Hilbert calculi (called Hilbert calculi with careful reasoning in Carnielli et al. (2008, Definition 2.3.1)) are of the form $H=\left\langle\Theta, R_{g}, R_{l}\right\rangle$ where $\Theta$ is a propositional signature and $R_{g} \cup R_{l}$ is a set of inference rules such that $R_{l} \subseteq R_{g}$ and no element of $R_{g} \backslash R_{l}$ is an axiom schema. Elements of $R_{g}$ and $R_{l}$ are called global and local inference rules, respectively. Given $\Gamma \cup\{\varphi\} \subseteq \operatorname{For}(\Theta), \varphi$ is globally derivable from $\Gamma$ in $H$, written $\Gamma \vdash_{H}^{g} \varphi$, if $\varphi$ is derivable from $\Gamma$ in the Hilbert calculus $\left\langle\Theta, R_{g}\right\rangle$ by using the standard definition (see Definition 6.2). On the other hand, in local derivations, besides using the local rules and the premises, global rules can be used provided that the premises are (global) theorems. In formal terms, $\varphi$ is locally derivable from $\Gamma$ in $H$, written $\Gamma \vdash_{H}^{l} \varphi$, if there exists a finite sequence of formulas $\varphi_{1} \ldots \varphi_{n}$ such that $\varphi_{n}$ is $\varphi$ and, for every $1 \leq i \leq n$, either $\varphi_{i} \in \Gamma$, or $\vdash_{H}^{g} \varphi_{i}$, or $\varphi_{i}$ is the consequence of some inference rule of $R_{l}$ whose premises appear in the sequence $\varphi_{1} \ldots \varphi_{i-1}$ (observe that this includes the case where $\varphi_{i}$ is an instance of an axiom in $R_{l}$ ). Obviously, local derivations are global derivations, but the converse does not necessarily hold: for instance, $\varphi \vdash_{H}^{g} \square \varphi$ always hold in a Hilbert calculus $H$ for a normal modal logic (say, $\mathbf{K}$ ), while $\varphi \vdash_{H}^{l} \square \varphi$ holds iff $\varphi$ is a theorem ${ }^{14}$ Clearly, local and global theorems coincide.

For instance, a Hilbert calculus for a (normal) modal logic typically contains, as local inference rules, (MP) and the axiom schemas, while the set of global rules is $\left(R_{l}\right)$ plus the

\footnotetext{
${ }^{14}$ This is clearer in terms of Kripke semantics: if $\varphi$ is a contingent formula, there is a Kripke model $M$ which satisfies $\varphi$ in a world $w$, but there may exist another world $w^{\prime}$ related to $w$ in which $M$ does not satisfy $\varphi$; hence, $M$ does not satisfy $\square \varphi$ in $w$.
} 
necessitation rule. As we have seen in Section 5.1, for the minimal non-normal modal logic $\mathbf{E}$ we have a similar case, with the replacement rule for $\square$ being employed instead of the necessitation rule. In this case, the deduction metatheorem only holds for local derivations. Note that, by definition, derivations in $\mathbf{R} \mathbf{m b C} \mathbf{C}^{*}$ lie in the scope of global derivations, while derivations in $\mathbf{R} \mathbf{m b C}$ are local derivations. Hence, the extension of $\mathbf{m b C}$ with replacement can be recast as a Hilbert calculus with careful reasoning $\mathbf{R m b C}^{+}=\left\langle\Sigma, R_{g}, R_{l}\right\rangle$ such that $R_{l}$ contains the axiom schemas of $\mathbf{m b C}$ plus (MP), and $R_{g}$ contains, besides this, the rules $\left(\mathbf{R}_{\neg}\right)$ and $\left(\mathbf{R}_{\circ}\right)$. Of course, the same can be done with the axiomatic extensions of $\mathbf{m b C}$ (and so of $\mathbf{R m b C}$ ) considered in Section 3 ,

At the semantical level, local derivations correspond to degree-preserving semantics w.r.t. a given class $\mathbb{M}$ of algebras, while global derivations correspond to truth-preserving semantics w.r.t. the class $\mathbb{M}$.

The presentation of LFIs with replacement as Hilbert calculi with careful reasoning (as the case of $\mathbf{R m b C}^{+}$) can be useful in order to combine these logics with (standard) normal modal logics by algebraic fibring: in this case, completeness of the fibring of the corresponding Hilbert calculi w.r.t. a semantics given by classes of suitable expansions of Boolean algebras would be immediate, according to the results stated in Carnielli et al. (2008, Chapter 2). By considering, as done in Zanardo et al. (2001), classes $\mathbb{M}$ of powerset algebras (i.e., with carrier of the form $\wp(W)$ for a non-empty set $W$ ) induced by Kripke models (which can be generalized to neighborhood models), then the fibring of, say, $\mathbf{R m b C}^{+}$with a given modal logic would simply be a minimal bimodal logic $\mathbf{E} \oplus \mathbf{E}$ with an additional modality $\square$, from which we derive the following modalities: $\nabla \varphi \stackrel{\text { def }}{=} \sim \square \sim \varphi, \neg \varphi \stackrel{\text { def }}{=} \varphi \rightarrow \square_{1} \varphi$, and $\circ \varphi \stackrel{\text { def }}{=} \sim\left(\varphi \wedge \square_{1} \varphi\right) \wedge \square_{2} \varphi$. This opens interesting opportunities for future research.

\section{Extension to first-order logics}

The next step is extending $\mathbf{R m b C}$, as well as its axiomatic extensions analyzed above, to firstorder languages. We begin by extending the notion of a propositional signature to the first-order case.

Definition 7.1 Let $\Sigma$ be a propositional signature. A first-order extension of $\Sigma$ is a firstorder signature $\Omega=\left\langle\Sigma, \mathcal{C},\left\{\mathcal{F}_{n}\right\}_{n \geq 1},\left\{\mathcal{P}_{n}\right\}_{n \geq 1}\right\rangle$ where:

- $\mathcal{C}$ is a denumerable set of individual constants;

- each $\mathcal{F}_{n}$ is a denumerable set of function symbols of arity $n$,

- each $\mathcal{P}_{n}$ is a denumerable set of predicate symbols of arity $n$.

The set $\mathcal{C}$ as well as any of $\mathcal{F}_{n}$ and $\mathcal{P}_{n}$ may be empty. However, $\bigcup_{n>1} \mathcal{P}_{n}$ must be nonempty. In the sequel, we work with a fixed denumerable set $\operatorname{Var}=\left\{v_{1}, v_{2}, \ldots\right\}$ of individual variables. The first-order language over $\Omega$ is defined as follows. The set of terms $\operatorname{Ter}(\Omega)$ (resp. closed terms $C \operatorname{Ter}(\Omega)$ ) is freely generated by $\operatorname{Var} \cup \mathcal{C}($ resp. $\mathcal{C})$ employing the elements of $\bigcup_{n \geq 1} \mathcal{F}_{n}$ as connectives. The set of atomic formulas $A F \operatorname{or}(\Omega)$ is composed of expressions of the form $P\left(\tau_{1}, \ldots, \tau_{n}\right)$ where $P \in \mathcal{P}_{n}$ and $\tau_{i} \in \operatorname{Ter}(\Omega)$ for $1 \leq i \leq n$. Finally, the set of formulas $\operatorname{For}_{1}(\Omega)$ is freely generated by $A F \operatorname{or}(\Omega)$ employing $\Sigma \cup\{\forall v\}_{v \in V a r} \cup\{\exists v\}_{v \in V a r}$ as connectives, where the latter two are $V a r$-indexed sets of quantifier symbols which we treat as having arity 1.

The set of closed formulas (or sentences) will be denoted by $\operatorname{Sen}(\Omega)$. The formula obtained from a given formula $\varphi$ by substituting every free occurrence of a variable $x$ by a term $t$ will be denoted by $\varphi[x / t]$.

Definition 7.2 Let $\Omega$ be a first-order signature extending $\Sigma$ from Definition 2.2. The logic $\mathbf{R Q m b C}$ is obtained from $\mathbf{R m b C}$ by adding the following axioms and rules: 


\section{Axiom Schemas:}

$$
\begin{aligned}
& (\mathbf{A} \mathbf{x} \exists) \quad \varphi[x / t] \rightarrow \exists x \varphi, \quad \text { if } t \text { is a term free for } x \text { in } \varphi \\
& (\mathbf{A} \mathbf{x} \forall) \quad \forall x \varphi \rightarrow \varphi[x / t], \text { if } t \text { is a term free for } x \text { in } \varphi
\end{aligned}
$$

\section{Inference rules:}

$$
\begin{aligned}
& \text { ( } \exists \text {-In) } \frac{\varphi \rightarrow \psi}{\exists x \varphi \rightarrow \psi}, \text { where } x \text { does not occur free in } \psi \\
& \left(\forall \text {-In) } \frac{\varphi \rightarrow \psi}{\varphi \rightarrow \forall x \psi}, \text { where } x \text { does not occur free in } \varphi\right.
\end{aligned}
$$

The consequence relation of RQmbC, suitably extending the one for $\mathbf{R m b C}$ (recall Definition 2.6 with the axioms and rules above, will be denoted by $\vdash_{\mathbf{R Q m b C}}$. Recall that a Hilbert calculus with careful reasoning for $\mathbf{R m b C}$ called $\mathbf{R} \mathbf{m b C}{ }^{+}$was defined at the end of Section 6 . This can be extended to the first-order case by considering the Hilbert calculus with careful reasoning $\mathbf{R Q m b C} \mathbf{C}^{+}$over a given first-order signature $\Omega$, such that $R_{l}$ contains (MP) and the axiom schemas of $\mathbf{m b C}$ plus $(\mathbf{A} \mathbf{x} \exists)$ and $(\mathbf{A} \mathbf{x} \forall)$. In turn, $R_{g}$ contains, besides the rules $R_{l}$, the (global) rules $\left(\mathbf{R}_{\neg}\right),\left(\mathbf{R}_{\circ}\right),(\exists-\mathbf{I n})$ and $(\forall-\mathbf{I n})$ (over $\Omega$ ). The property of replacement for $\mathbf{R Q m b C}$ follows directly from the propositional case (Theorem 2.8), observing that quantifiers (seen as unary operators) clearly preserve logical equivalence.

TheOREM 7.3 Let $\mathbf{L}$ be the logic $\mathbf{R Q m b C}$ or any axiomatic extension of it over the signature $\Omega$, in which the derivation from premises is defined as in $\mathbf{R Q m b C}$. Then, $\mathbf{L}$ satisfies the replacement property.

Given that, as in the case of $\mathbf{R m b C}, \mathbf{R Q m b C}$ uses local reasoning, it satisfies the deduction metatheorem without any restrictions. This is different from what happens with $\mathbf{Q m b C}$, a firstorder version of $\mathbf{m b C}$ proposed in Carnielli et al. (2014), where this metatheorem holds with the same restrictions as in first-order classical logic 15

Theorem 7.4 (Deduction Metatheorem for RQmbC) $\Gamma, \varphi \vdash_{\mathbf{R Q m b C}} \psi$ if and only if $\Gamma \vdash_{\mathbf{R Q m b C}} \varphi \rightarrow \psi$.

\subsection{BALFI semantics for RQmbC}

In Coniglio et al. (2020) a semantics of first-order structures, based on swap structures over complete Boolean algebras, was obtained for $\mathbf{Q m b C}$. Since RQmbC is self-extensional, that semantics can be drastically simplified, and so the non-deterministic swap structures will be replaced by BALFIs, which are ordinary algebras. The semantics will be defined in an analogous fashion as we did for the propositional case, where valuations can be considered as homomorphisms between the algebra of formulas and BALFIs. In the sequel, only BALFIs over complete Boolean algebras will be considered. This requirement is justified since quantifiers will be interpreted by means of (infinite) infima and suprema in Boolean algebras, hence their existence must be always guaranteed.

\footnotetext{
${ }^{15}$ It is worth mentioning that the only difference between $\mathbf{Q m b C}$ and $\mathbf{R Q m b C}$ is that the latter contains the inference rules $\left(\mathbf{R}_{\neg}\right)$ and $\left(\mathbf{R}_{\circ}\right)$, which are not present in the former (besides the different notions of derivation from premises adopted in $\mathbf{Q m b C}$ and in $\mathbf{R Q m b C}$ ). In addition, da Costa's axioms included in $\mathbf{Q m b C}$, which state the equivalence between two formulas such that one is a variant of the other (that is, they coincide up to void quantifiers and renaming of bounded variables), is no longer necessary in RQmbC.
} 
Definition $7.5 A$ Boolean algebra $\mathcal{B}$ is complete if there exist in $\mathcal{B}$ both the infimum $\bigwedge X$ and the supremum $\bigvee X$ of every subset $X$ of its carrier. A complete BALFI is a BALFI such that its reduct to $\Sigma_{B A}$ is a complete Boolean algebra.

Definition 7.6 Let $\Omega$ be a first-order signature. Moreover, let $\mathcal{B}$ be a complete BALFI with carrier $A$ and $U$ be some nonempty set (called universe or domain). A (first-order) structure (or a $\mathbf{R Q m b C - s t r u c t u r e ) ~ o v e r ~} \Omega$ is a tuple $\mathfrak{A}=\left\langle U, \mathcal{B}, I_{\mathfrak{A}}\right\rangle$ such that $I_{\mathfrak{A}}$ is an interpretation function which assigns:

- an element $I_{\mathfrak{A}}(c)$ of $U$ to each individual constant $c \in \mathcal{C}$;

- a function $I_{\mathfrak{A}}(f): U^{n} \rightarrow U$ to each function symbol $f$ of arity $n$;

- a function $I_{\mathfrak{A}}(P): U^{n} \rightarrow A$ to each predicate symbol $P$ of arity $n$.

From now on, we will write $c^{\mathfrak{A}}, f^{\mathfrak{A}}$ and $P^{\mathfrak{A}}$ instead of $I_{\mathfrak{A}}(c), I_{\mathfrak{A}}(f)$ and $I_{\mathfrak{A}}(P)$ to denote the interpretation of an individual constant symbol $c$, a function symbol $f$ and a predicate symbol $P$, respectively.

Definition 7.7 Given a structure $\mathfrak{A}=\left\langle U, \mathcal{B}, I_{\mathfrak{A}}\right\rangle$ over $\Omega$, an assignment over $\mathfrak{A}$ is any function $\mu: \operatorname{Var} \rightarrow U$. The set of assignments over $\mathfrak{A}$ will be denoted by $\mathrm{A}(\mathfrak{A})$. For every $a \in U$ and every assignment $\mu$, let $\mu_{a}^{x}$ be the assignment such that $\mu_{a}^{x}(x)=a$ and $\mu_{a}^{x}(y)=\mu(y)$ if $y \neq x$.

Definition 7.8 (Term denotation) Given a structure $\mathfrak{A}$ over $\Omega$, and given an assignment $\mu$ over $\mathfrak{A}$, we define recursively, for each term $t$, an element $\llbracket t \rrbracket_{\mu}^{\mathfrak{A}}$ in $U$ as follows:

- $\llbracket c \rrbracket_{\mu}^{\mathfrak{A}}=c^{\mathfrak{A}}$ if $c$ is an individual constant;

- $\llbracket x \rrbracket_{\mu}^{\mathfrak{A}}=\mu(x)$ if $x$ is a variable;

- $\llbracket f\left(t_{1}, \ldots, t_{n}\right) \rrbracket_{\mu}^{\mathfrak{A}}=f^{\mathfrak{A}}\left(\llbracket t_{1} \rrbracket_{\mu}^{\mathfrak{A}}, \ldots, \llbracket t_{n} \rrbracket_{\mu}^{\mathfrak{A}}\right)$ if $f$ is a function symbol of arity $n$ and $t_{1}, \ldots, t_{n}$ are terms.

Definition 7.9 (RQmbC interpretation maps) Let $\mathcal{B}$ be a complete BALFI and $\mathfrak{A}=\left\langle U, \mathcal{B}, I_{\mathfrak{A}}\right\rangle$ a structure over $\Omega$. The interpretation map for $\mathbf{R Q m b C}$ over $\mathfrak{A}$ is a function $\llbracket \cdot \rrbracket_{\mu}^{\mathfrak{A}}:$ For $_{1}(\Omega) \times$ $\mathrm{A}(\mathfrak{A}) \rightarrow A$ (for a given assigment $\mu$ over $\mathfrak{A}$ ) satisfying the following clauses:

(i) $\quad \llbracket P\left(t_{1}, \ldots, t_{n}\right) \rrbracket_{\mu}^{\mathfrak{A}}=P^{\mathfrak{A}}\left(\llbracket t_{1} \rrbracket_{\mu}^{\mathfrak{A}}, \ldots, \llbracket t_{n} \rrbracket_{\mu}^{\mathfrak{A}}\right)$, for $P\left(t_{1}, \ldots, t_{n}\right)$ atomic;

(ii) $\quad \llbracket \# \varphi \rrbracket_{\mu}^{\mathfrak{A}}=\# \llbracket \varphi \rrbracket_{\mu}^{\mathfrak{A}}, \quad$ for every $\# \in\{\neg, \circ\}$;

(iii) $\llbracket \varphi \# \psi \rrbracket_{\mu}^{\mathfrak{A}}=\llbracket \varphi \rrbracket_{\mu}^{\mathfrak{A}} \# \llbracket \psi \rrbracket_{\mu}^{\mathfrak{A}}, \quad$ for every $\# \in\{\wedge, \vee, \rightarrow\}$;

(iv) $\quad \forall x \varphi \rrbracket_{\mu}^{\mathfrak{A}}=\bigwedge\left\{\llbracket \varphi \rrbracket_{\nu}^{\mathfrak{A}}: \nu=\mu_{a}^{x} ; a \in U\right\} ;$

(v) $\quad \llbracket \exists x \varphi \rrbracket_{\mu}^{\mathfrak{A}}=\bigvee\left\{\llbracket \varphi \rrbracket_{\nu}^{\mathfrak{A}}: \nu=\mu_{a}^{x} ; a \in U\right\}$.

REMARK 7.10

(1) If $t$ is a closed term we can write $\llbracket t \rrbracket^{\mathfrak{A}}$ instead of $\llbracket t \rrbracket_{\mu}^{\mathfrak{A}}$, for any assignment $\mu$, since it does not depend on $\mu$. Analogously, we can write $\llbracket \varphi \rrbracket^{\mathfrak{A}}$ instead of $\llbracket \varphi \rrbracket_{\mu}^{\mathfrak{A}}$ for a closed formula $\varphi$.

(2) The last two items can equivalently be written as:

$$
\left(i v^{*}\right) \llbracket \forall x \varphi \rrbracket_{\mu}^{\mathfrak{A}}=\bigwedge_{a \in U} \llbracket \varphi \rrbracket_{\mu_{a}^{x}}^{\mathfrak{A}} \quad \text { and } \quad\left(v^{*}\right) \llbracket \exists x \varphi \rrbracket_{\mu}^{\mathfrak{A}}=\bigvee_{a \in U} \llbracket \varphi \rrbracket_{\mu_{a}^{x}}^{\mathfrak{A}}
$$

If $x$ does not appear free in $\varphi$ we have that $\llbracket \forall x \varphi \rrbracket_{\mu}^{\mathfrak{A}}=\llbracket \varphi \rrbracket_{\mu}^{\mathfrak{A}}$ and $\llbracket \exists x \varphi \rrbracket_{\mu}^{\mathfrak{A}}=\llbracket \varphi \rrbracket_{\mu}^{\mathfrak{A}}$.

Definition 7.11 Let $\mathfrak{A}$ be a RQmbC-structure over $\Omega$. A formula $\varphi$ in For $_{1}(\Omega), \varphi$ is said to be valid in $\mathfrak{A}$, denoted by $\models_{\mathfrak{A}} \varphi$, if $\llbracket \varphi \rrbracket_{\mu}^{\mathfrak{A}}=1$, for every assignment $\mu$. We say that $\varphi$ is valid in $\mathbf{R Q m b C}$, denoted by $\models_{\mathbf{R Q m b C}} \varphi$, if $\models_{\mathfrak{A}} \varphi$, for every $\mathfrak{A}$. 
DeFINITIOn 7.12 (First-order degree-preserving BALFI semantics) Let $\Gamma \cup\{\varphi\}$ be a set of formulas in $\operatorname{For}_{1}(\Omega)$. Then $\varphi$ is said to be a semantical consequence of $\Gamma$ in $\mathbf{R Q m b C}$ w.r.t. BALFIs, denoted by $\Gamma \models_{\mathbf{R Q m b C}} \varphi$, if either $\varphi$ is valid in $\mathbf{R Q m b C}$, or there exists a finite, non-empty subset $\left\{\gamma_{1}, \ldots, \gamma_{n}\right\}$ of $\Gamma$ such that the formula $\left(\gamma_{1} \wedge \ldots \wedge \gamma_{n}\right) \rightarrow \varphi$ is valid in RQmbC.

In order to prove the soundness of $\mathbf{R Q m b C}$ w.r.t. BALFI semantics, it is necessary to state an important result:

TheOREM 7.13 (Substitution Lemma) Let $\mathcal{B}$ be a complete BALFI, $\mathfrak{A}$ a structure over $\Omega$, and $\mu$ an assignment over $\mathfrak{A}$. If $t$ is a term free for $z$ in $\varphi$ and $b=\llbracket t \rrbracket_{\mu}^{\mathfrak{A}}$, then $\llbracket \varphi[z / t] \rrbracket_{\mu}^{\mathfrak{A}}=\llbracket \varphi \rrbracket_{\mu_{b}^{z}}^{\mathfrak{A}}$.

Proof. The result is easily proved by induction on the complexity of $\varphi$.

THEOREM 7.14 (Soundness of RQmbC w.r.t. BALFIs)

For every set $\Gamma \cup\{\varphi\} \subseteq$ For $_{1}(\Omega): \Gamma \vdash_{\mathbf{R Q m b C}} \varphi$ implies that $\Gamma \models \mathbf{R Q m b C} \varphi$.

Proof. It will be proven by extending the proof of soundness of $\mathbf{R m b C}$ w.r.t. BALFI semantics (Theorem 2.14). Thus, the only cases required to be analyzed are the new axioms and inference rules. By the very definitions, and taking into account Theorem 7.13 , it is immediate to see that axioms $(\mathbf{A} \mathbf{x} \exists)$ and $(\mathbf{A} \mathbf{x} \forall)$ are valid in any $\mathfrak{A}$. With respect to $(\exists$-In $)$, suppose that $\alpha \rightarrow \beta$ is valid in a given $\mathfrak{A}$, where the variable $x$ does not occur free in $\beta$. Then $\llbracket \alpha \rrbracket_{\mu}^{\mathfrak{A}} \leq \llbracket \beta \rrbracket_{\mu}^{\mathfrak{A}}$ for every assignment $\mu$. In particular, for every $a \in U, \llbracket \alpha \rrbracket_{\mu_{a}^{x}}^{\mathfrak{A}} \leq \llbracket \beta \rrbracket_{\mu_{a}^{x}}^{\mathfrak{A}}=\llbracket \beta \rrbracket_{\mu}^{\mathfrak{A}}$, since $x$ is not free in $\beta$. But then: $\llbracket \exists x \alpha \rrbracket_{\mu}^{\mathfrak{A}}=\bigvee_{a \in U} \llbracket \alpha \rrbracket_{\mu_{a}^{x}}^{\mathfrak{A}} \leq \llbracket \beta \rrbracket_{\mu}^{\mathfrak{A}}$. Hence, $\exists x \alpha \rightarrow \beta$ is valid in $\mathfrak{A}$. The case for $(\forall-\mathbf{I n})$ is proved analogously.

\subsection{Completeness of RQmbC w.r.t. BALFI semantics}

Along this section, in which the completeness of RQmbC w.r.t. BALFI semantics will be shown, $\Omega$ will denote a fixed first-order signature, over which the consequence relations $\vdash_{\mathbf{R Q m b C}}$ and $\models_{\mathbf{R Q m b C}}$ are defined. In turn, $\Omega_{C}$ will denote the signature obtained from $\Omega$ by adding an infinite and denumerable set $C$ of new individual constants. The syntactical consequence relation of $\mathbf{R Q m b C}$ over the signature $\Omega_{C}$ will be denoted by $\vdash_{\mathbf{R Q m b C}}^{C}$.

Proposition 7.15 If $\Gamma \cup\{\varphi\} \in$ For $_{1}(\Omega)$, then: $\Gamma \vdash_{\mathbf{R Q m b C}}^{C} \varphi$ iff $\Gamma \vdash_{\mathbf{R Q m b C}} \varphi$.

Proof. Observe that it is enough to prove the case for $\Gamma=\emptyset$, since the general case follows easily from this. The 'if' part is an immediate consequence of the definition of $\vdash_{\mathbf{R Q m b C}}^{C}$. Now, assume that $\vdash_{\mathbf{R Q m b C}}^{C} \varphi$, for $\varphi \in \operatorname{For}_{1}(\Omega)$, and let $d$ be a derivation $\varphi_{1} \ldots \varphi_{n}$ of $\varphi$ in $\mathbf{R Q m b C}$ over the signature $\Omega_{C}$ (hence $\varphi_{n}=\varphi$ ). Since the derivation $d$ is finite and the length of the formulas is also finite, the set of constants in $C$ occurring in $d$ is contained in $\left\{c_{1}, \ldots, c_{m}\right\}$ for some $m \geq 1$. Let $x_{1}, \ldots, x_{m}$ be $m$ different fresh variables, that is, $x_{i} \neq x_{j}$ if $i \neq j$, and no $x_{i}$ occurs (free or bounded) in the formulas of the derivation $d$. Let $\varphi_{i}^{\prime}$ be the formula obtained from $\varphi_{i}$ by substituting uniformly the constant $c_{j}$ by the variable $x_{j}$, for $1 \leq i \leq n$ and $1 \leq j \leq m$. Observe that $\varphi_{m}^{\prime}=\varphi$. Then, it is clear that the sequence $d^{\prime}$ given by $\varphi_{1}^{\prime} \ldots \varphi_{n}^{\prime}$ is a derivation of $\varphi$ in RQmbC over the signature $\Omega$. From this, $\vdash_{\mathbf{R Q m b C}} \varphi$.

By an easy adaptation of the first part of the proof of Theorem 2.15 the following can be proved:

Proposition 7.16 Let $\equiv^{C} \subseteq$ For $_{1}\left(\Omega_{C}\right)^{2}$ be a relation in For ${ }_{1}\left(\Omega_{C}\right)$ defined as follows: $\alpha \equiv^{C} \beta$ iff $\vdash{ }_{\mathbf{R Q m b C}}^{C} \alpha \leftrightarrow \beta$. Then, $\equiv^{C}$ is a congruence over For $_{1}\left(\Omega_{C}\right)$ (seen as an algebra over $\Sigma$ ) such that the operations $[\alpha]^{C} \#[\beta]^{C} \stackrel{\text { def }}{=}[\alpha \# \beta]^{C}$ for any $\# \in\{\wedge, \vee, \rightarrow\}, \#[\alpha]^{C} \stackrel{\text { def }}{=}[\# \alpha]^{C}$ for any $\# \in\{\neg, \circ\}, 0^{C} \stackrel{\text { def }}{=}[\alpha \wedge(\neg \alpha \wedge \circ \alpha)]^{C}$ and $1^{C} \stackrel{\text { def }}{=}[\alpha \vee \neg \alpha]^{C}$ (where $[\alpha]^{C}$ denotes the equivalence class of $\alpha$ w.r.t. $\left.\equiv^{C}\right)$ define a BALFI $\mathcal{B}_{C}^{0}$ with carrier $A_{C} \stackrel{\text { def }}{=}$ For $_{1}\left(\Omega_{C}\right) / \equiv^{C}$. 
The Boolean algebra underlying the BALFI $\mathcal{B}_{C}^{0}$ will be denoted by $\mathcal{A}_{C}$.

The construction of the canonical model for RQmbC requires a complete BALFI, hence the Boolean algebra $\mathcal{A}_{C}$ must be completed. Recall ${ }^{16}$ that a Boolean algebra $\mathcal{A}^{\prime}$ is a completion of a Boolean algebra $\mathcal{A}$ if: (1) $\mathcal{A}^{\prime}$ is complete, and (2) $\mathcal{A}^{\prime}$ includes $\mathcal{A}$ as a dense subalgebra (that is: every element in $A^{\prime}$ is the supremum, in $\mathcal{A}^{\prime}$, of some subset of $A$ ). From this, $\mathcal{A}^{\prime}$ preserves all the existing infima and suprema in $\mathcal{A}$. In formal terms: there exists a monomorphism of Boolean algebras (therefore an injective mapping) $*: \mathcal{A} \rightarrow \mathcal{A}^{\prime}$ such that $*\left(\bigvee_{\mathcal{A}} X\right)=\bigvee_{\mathcal{A}^{\prime}} *[X]$ for every $X \subseteq A$ such that the supremum $\bigvee_{\mathcal{A}} X$ exists, where $*[X]=\{*(a): a \in X\}$. Analogously, $*\left(\bigwedge_{\mathcal{A}} X\right)=\bigwedge_{\mathcal{A}^{\prime}} *[X]$ for every $X \subseteq A$ such that the infimum $\bigwedge_{\mathcal{A}} X$ exists. By well-known results obtained independently in MacNeille (1937) and Tarski (1937), it follows that every Boolean algebra has a completion; moreover, the completion is unique up to isomorphisms. Based on this, let $\mathrm{C} \mathcal{A}_{C}$ be the completion of $\mathcal{A}_{C}$ and let $*: \mathcal{A}_{C} \rightarrow \mathrm{C} \mathcal{A}_{C}$ be the associated monomorphism. Recalling from Proposition 7.16 that $\mathcal{A}_{C}$ is the reduct of the BALFI $\mathcal{B}_{C}^{0}$, we can define the following:

Definition 7.17 Let $\mathcal{C A}_{C}$ be the complete Boolean algebra defined as above. The canonical $B A L F I$ for $\mathbf{R Q m b C}$ over $\Omega_{C}$, denoted by $\mathcal{B}_{C}$, is obtained from $C_{\mathcal{A}}$ by adding the unary operators $\neg_{*}$ and $\circ_{*}$ defined as follows: $\neg_{*} b=*(\neg a)$ if $b=*(a)$, and $\neg_{*} b=\sim b$ if $b \notin *\left[A_{C}\right]$ (where $\sim$ is the Boolean complement in $\mathrm{CA}_{C}$ and $\neg$ is the operation in $\left.\mathcal{B}_{C}^{0}\right) ; \circ_{*} b=*(\circ a)$ if $b=*(a)$, and $\circ_{*} b=1$ if $b \notin *\left[A_{C}\right]$ (where $*$ is the operation in $\left.\mathcal{B}_{C}^{0}\right){ }^{17}$

Proposition 7.18 The operations over $\mathcal{B}_{C}$ are well-defined, and $\mathcal{B}_{C}$ is a complete BALFI such that $*\left([\alpha]^{C}\right)=1$ iff $\vdash_{\mathbf{R Q m b C}}^{C} \alpha$.

Proof. Since $*\left[A_{C}\right]$ is a subalgebra of $\mathrm{CA}_{C}, b \notin *\left[A_{C}\right]$ iff $\sim b \notin *\left[A_{C}\right]$. On the other hand, $*$ is injective. This shows that $\neg_{*}$ and $\circ_{*}$ are well-defined. The rest of the proof is obvious from the definitions.

Definition 7.19 (Canonical Structure) Let $\mathcal{B}_{C}$ be as in Definition 7.17, and let $U=C T e r\left(\Omega_{C}\right)$. The canonical structure induced by $C$ is the structure $\mathfrak{A}_{C}=\left\langle U, \mathcal{B}_{C}, I_{\mathfrak{A}_{C}}\right\rangle$ over $\Omega_{C}$ such that:

$-c^{\mathfrak{A}_{C}}=c$, for each individual constant $c$;

- $f^{\mathfrak{A}_{C}}: U^{n} \rightarrow U$ is such that $f^{\mathfrak{A}_{C}}\left(t_{1}, \ldots, t_{n}\right)=f\left(t_{1}, \ldots, t_{n}\right)$, for each $n$-ary function symbol $f$;

- $P^{\mathfrak{A}_{C}}\left(t_{1}, \ldots, t_{n}\right)=*\left(\left[P\left(t_{1}, \ldots, t_{n}\right)\right]^{C}\right)$, for each $n$-ary predicate symbol $P$.

Lemma 7.20 Let $\mathcal{A}_{C}$ be the Boolean algebra obtained as in Proposition 17.16, and let $U=$ $C T e r\left(\Omega_{C}\right)$. Then, for every formula $\psi(x)$ in For $_{1}\left(\Omega_{C}\right)$ with (at most) a free variable $x$ it holds: (1) $[\forall x \psi]^{C}=\bigwedge_{\mathcal{A}_{C}}\left\{[\psi[x / t]]^{C}: t \in U\right\}$, where $\bigwedge_{\mathcal{A}_{C}}$ denotes an existing infimum in the Boolean algebra $\mathcal{A}_{C}$;

(2) $[\exists x \psi]^{C}=\bigvee_{\mathcal{A}_{C}}\left\{[\psi[x / t]]^{C}: t \in U\right\}$, where $\bigvee_{\mathcal{A}_{C}}$ denotes an existing supremum in the Boolean algebra $\mathcal{A}_{C}$.

Proof.

(1) By the given definitions and by the rules from $\mathbf{C P L}^{+},[\alpha]^{C} \leq[\beta]^{C}$ in $\mathcal{A}_{C}$ iff $\vdash_{\mathbf{R Q m b C}}^{C} \alpha \rightarrow \beta$. Let $\psi(x)$ be a formula in $\operatorname{For}_{1}\left(\Omega_{C}\right)$ with (at most) a free variable $x$. Then $[\forall x \psi]^{C} \leq[\psi[x / t]]^{C}$ for every $t \in U$, by $(\mathbf{A} \mathbf{x} \forall)$. Let $\beta$ be a formula in $\operatorname{For}_{1}\left(\Omega_{C}\right)$ such that $[\beta]^{C} \leq[\psi[x / t]]^{C}$ for

\footnotetext{
${ }^{16}$ See, for instance, Givant and Halmos (2009, Chapter 25).

${ }^{17}$ Observe that the definition of the operations $\neg_{*}$ and $\circ_{*}$ outside the image of $*$ meet the requirements of BALFIs. Of course other options, coherent with the definition of BALFIs, could be possible for such values outside $*\left[A_{C}\right]$, still defining a BALFI structure over $C \mathcal{A}_{C}$.
} 
every $t \in U$. Given that $\beta$ and $\psi(x)$ are finite expressions and $C$ is infinite, there exists a constant $c$ in $C$ such that $c$ neither occurs in $\beta$ nor in $\psi(x)$ and, by hypothesis, $[\beta]^{C} \leq[\psi[x / c]]^{C}$. That is, $\vdash_{\mathbf{R Q m b C}}^{C} \beta \rightarrow \psi[x / c]$. Let $d$ be a derivation $\varphi_{1} \ldots \varphi_{n}$ of $\beta \rightarrow \psi[x / c]$ in RQmbC over the signature $\Omega_{C}$ (hence $\varphi_{n}=\beta \rightarrow \psi[x / c]$ ), and let $z$ be a variable which does not occur (free or bounded) in the formulas of the derivation $d$. Observe that such variable always exists, since the number of variables occurring (free or bounded) in $d$ is finite. Let $\varphi_{i}^{\prime}$ be the formula obtained from $\varphi_{i}$ by substituting uniformly the constant $c$ by the variable $z$, for $1 \leq i \leq n$. Observe that $\varphi_{n}^{\prime}=\beta \rightarrow \psi[x / z]$, given that $c$ occurs neither in $\beta$ nor in $\psi(x)$. Since $z$ does not occur in $d$, it is free for $x$ in $\psi$ and it does not occur in $\beta$. By an argument similar to the one given in the proof of Proposition 7.15, the sequence $d^{\prime}$ given by $\varphi_{1}^{\prime} \ldots \varphi_{n}^{\prime}$ is a derivation of $\beta \rightarrow \psi[x / z]$ in RQmbC over the signature $\Omega_{C}$, such that $z$ does not occur free in $\beta$. By applying the rule $\left(\forall\right.$-In), it follows that $\vdash_{\mathbf{R Q m b C}}^{C} \beta \rightarrow \forall z \psi[x / z]$. But $z$ is free for $x$ in $\psi$, hence $\vdash_{\mathbf{R Q m b C}}^{C} \forall z \psi[x / z] \rightarrow \forall x \psi$ and so $\vdash_{\mathbf{R Q m b C}}^{C} \beta \rightarrow \forall x \psi$. That is, $[\beta]^{C} \leq[\forall x \psi]^{C}$. This shows that $[\forall x \psi]^{C}=\bigwedge_{\mathcal{A}_{C}}\left\{[\psi[x / t]]^{C}: t \in U\right\}$.

(2) It is proved analogously.

Proposition 7.21 For every sentence $\psi$ in $\operatorname{Sen}\left(\Omega_{C}\right)$, $\llbracket \psi \rrbracket^{\mathfrak{A}_{C}}=*\left([\psi]^{C}\right)$. Moreover, $\llbracket \psi \rrbracket^{\mathfrak{A}_{C}}=$ $1^{C}$ iff $\vdash_{\mathbf{R Q m b C}}^{C} \psi$.

Proof. The proof is done by induction on the complexity of the sentence $\psi$ in $\operatorname{Sen}\left(\Omega_{C}\right)$. If $\psi=P\left(t_{1}, \ldots, t_{n}\right)$ is atomic then, by using Definition 7.9 , the fact that $\llbracket t \rrbracket^{\mathfrak{A}_{C}}=t$ for every $t \in C \operatorname{Ter}\left(\Omega_{C}\right)$, and Definition 7.19, we have:

$\llbracket \psi \rrbracket^{\mathfrak{A}_{C}}=P^{\mathfrak{A}_{C}}\left(\llbracket t_{1} \rrbracket^{\mathfrak{A}_{C}}, \ldots, \llbracket t_{n} \rrbracket^{\mathfrak{A}_{C}}\right)=P^{\mathfrak{A}_{C}}\left(t_{1}, \ldots, t_{n}\right)=*\left([\psi]^{C}\right)$.

If $\psi=\# \beta$ for $\# \in\{\neg, \circ\}$ then, by Definitions 7.9 and 7.17 and by induction hypothesis,

$$
\llbracket \psi \rrbracket^{\mathfrak{A}_{C}}=\# * \llbracket \beta \rrbracket^{\mathfrak{A}_{C}}=\#_{*}\left(*\left([\beta]^{C}\right)\right)=*\left([\# \beta]^{C}\right) .
$$

If $\psi=\alpha \# \beta$ for $\# \in\{\wedge, \vee, \rightarrow\}$, the proof is analogous.

If $\psi=\forall x \beta$ then, by Lemma 7.20 and using that $U=C \operatorname{Ter}\left(\Omega_{C}\right),[\forall x \beta]^{C}=\bigwedge_{\mathcal{A}_{C}}\left\{[\beta[x / t]]^{C}\right.$ : $t \in U\}$ and so $*\left([\forall x \beta]^{C}\right)=\bigwedge_{\mathcal{C A}_{C}}\left\{*\left([\beta[x / t]]^{C}\right): t \in U\right\}$. Let $\mu$ be an assignment over $\mathfrak{A}_{C}$. Since $\llbracket t \rrbracket_{\mu}^{\mathfrak{A}_{C}}=t$ for every $t \in U$ it follows, by Theorem 7.13 and by induction hypothesis, that $\llbracket \beta \rrbracket_{\mu_{t}^{x}}^{\mathfrak{A}_{C}}=\llbracket \beta[x / t] \rrbracket_{\mu}^{\mathfrak{A}_{C}}=\llbracket \beta[x / t] \rrbracket^{\mathfrak{A}_{C}}=*\left([\beta[x / t]]^{C}\right)$. Then, by Definition 7.9 ;

$$
\llbracket \forall x \beta \rrbracket^{\mathfrak{A}_{C}}=\llbracket \forall x \beta \rrbracket_{\mu}^{\mathfrak{A}_{C}}=\bigwedge_{t \in U} \llbracket \beta \rrbracket_{\mu_{t}^{x}}^{\mathfrak{A}_{C}}=\bigwedge_{t \in U} *\left([\beta[x / t]]^{C}\right)=*\left([\forall x \beta]^{C}\right) .
$$

If $\psi=\exists x \beta$, the proof is analogous to the previous case.

This shows that $\llbracket \psi \rrbracket^{\mathfrak{A}_{C}}=*\left([\psi]^{C}\right)$ for every sentence $\psi$. The rest of the proof follows by Proposition 7.18 .

THEOREM 7.22 (Completeness of RQmbC w.r.t. BALFI semantics) For every $\Gamma \cup\{\varphi\} \subseteq \operatorname{Sen}(\Omega)$ : if $\Gamma \models \mathbf{R Q m b C} \varphi$ then $\Gamma \vdash_{\mathbf{R Q m b C}} \varphi$.

Proof. Let $\mathfrak{A}_{C}$ be as in Definition 7.19, and let $\mathfrak{A}^{\prime}$ be the reduct of $\mathfrak{A}_{C}$ to $\Omega{ }^{18}$ Let $\Gamma \cup\{\varphi\} \subseteq$ $\operatorname{Sen}(\Omega)$ such that $\Gamma \models \mathbf{R Q m b C} \varphi$. By Definition 7.11 , we have two cases to analyze:

(1) $\varphi$ is valid in $\mathbf{R Q m b C}$. Then, $\varphi$ is valid in $\mathfrak{A}^{\prime}$. It is routine to prove that, if $\psi \in \operatorname{For}_{1}(\Omega)$ and $\mu \in \mathrm{A}\left(\mathfrak{A}^{\prime}\right)=\mathrm{A}\left(\mathfrak{A}_{C}\right)$, then $\llbracket \psi \rrbracket_{\mu}^{\mathfrak{A}^{\prime}}=\llbracket \psi \rrbracket_{\mu}^{\mathfrak{A}_{C}}$. From this, $\varphi$ is valid in $\mathfrak{A}_{C}$. By Definition 7.11 $\llbracket \varphi \rrbracket^{\mathfrak{A}_{C}}=1^{C}$. Hence, $\vdash_{\mathbf{R Q m b C}} \varphi$, by Propositions 7.21 and 7.15 . Therefore, $\Gamma \vdash_{\mathbf{R Q m b C}} \varphi$, by

\footnotetext{
${ }^{18}$ That is, $\mathfrak{A}^{\prime}$ coincides with $\mathfrak{A}_{C}$, with the exception that it 'forgets' to interpret the constants in $C$. Hence, $\mathfrak{A}^{\prime}$ is a structure over $\Omega$.
} 
definition of derivations from premises in RQmbC.

(2) There exists a finite, non-empty subset $\left\{\gamma_{1}, \ldots, \gamma_{n}\right\}$ of $\Gamma$ such that the closed formula $\psi=$ $\left(\gamma_{1} \wedge \gamma_{2} \wedge \ldots \wedge \gamma_{n}\right) \rightarrow \varphi$ over $\Omega$ is valid in RQmbC. In particular, $\psi$ is valid in $\mathfrak{A}^{\prime}$ and so, as observed in (1), it is valid in $\mathfrak{A}_{C}$. But then, by item (1), we have that $\vdash_{\mathbf{R Q m b C}} \psi$, which implies that $\Gamma \vdash_{\mathbf{R Q m b C}} \varphi$, by definition of derivations from premises in $\mathbf{R Q m b C}$.

This completes the proof.

REMARK 7.23 The completeness result for $\mathbf{R Q m b C}$ w.r.t. BALFI semantics was obtained just for sentences, and not for formulas possibly containing free variables (as it was done with the soundness Theorem 7.14). This can be easily overcome. Recall that the universal closure of a formula $\psi$ in $\operatorname{For}_{1}(\Omega)$, denoted by $(\forall) \psi$, is defined as follows: if $\psi$ is a sentence then $(\forall) \psi \stackrel{\text { def }}{=} \psi$; and if $\psi$ has exactly the variables $x_{1}, \ldots, x_{n}$ occurring free then $(\forall) \psi \stackrel{\text { def }}{=} \forall x_{1} \cdots \forall x_{n} \psi$. If $\Gamma$ is a set of formulas in $\operatorname{For}_{1}(\Omega)$ then $(\forall) \Gamma \stackrel{\text { def }}{=}\{(\forall) \psi: \psi \in \Gamma\}$. It is easy to show that, for every $\Gamma \cup\{\varphi\} \subseteq$ For $_{1}(\Omega)$ : (i) $\Gamma \vdash_{\mathbf{R Q m b C}} \varphi$ iff $(\forall) \Gamma \vdash_{\mathbf{R Q m b C}}(\forall) \varphi$; and (ii) $\Gamma \models{ }_{\mathbf{R Q m b C}} \varphi$ iff $(\forall) \Gamma \models \mathbf{R Q m b C}(\forall) \varphi$. From this, a general completeness for $\mathbf{R Q m b C}$ result follows from Theorem 7.22.

\section{Conclusion, and significance of the results}

This paper offers a solution to two open problems in the domain of paraconsistency, in particular connected to algebraization of LFIs. The quest for the algebraic counterpart of paraconsistency is more than 50 years old: since the outset of da Costa's paraconsistent calculi, algebraic equivalents for such systems have been sought, with different degrees of success (and failure). Our results suggest that the new concepts and methods proposed in the present paper, in particular the neighborhood-style semantics introduced for BALFIs, have a good potential for applications. As suggested in Marcos (2005a), modal logics could alternatively be regarded as the study of a kind of modal-like contradiction-tolerant systems. In alternative to founding modal semantics in terms of belief, knowledge, tense, etc., modal logic could be regarded as a general 'theory of opposition', more akin to the Aristotelian tradition.

Applications of paraconsistent logics in computer science, probability and AI, just to mention a few areas, are greatly advanced when more traditional algebraic tools pertaining to extensions of Boolean algebras and neighborhood semantics, are used to express the underlying ideas of paraconsistency. In addition, many logical systems employed in deontic logic and normative reasoning, where non-normal modal logics and neighborhood semantics play an important role, could be extended by means of our approach. Hopefully, our results may unlock new research in this direction. Finally, BALFI semantics for LFIs opens the possibility of obtaining new algebraic models for paraconsistent set theory (Carnielli and Coniglio, 2016b, 2021) by generalizing the well-known Boolean-valued models for ZF (Bell, 2005).

Acknowledgements: The first and second authors acknowledge support from the National Council for Scientific and Technological Development $(\mathrm{CNPq})$, Brazil under research grants 307376/2018-4 and 306530/2019-8, respectively. The third author acknowledges support from the Luxembourg National Research Fund (FNR), grant CORE AuReLeE - Automated Reasoning with Legal Entities (C20/IS/14616644). 


\section{References}

Arruda, A. I. and N. C. A. da Costa (1970). Sur le schéma de la séparation. Nagoya Mathematical Journal 38, 71-84.

Avron, A. (2005). Non-deterministic matrices and modular semantics of rules. In J.-Y. Béziau (Ed.), Logica Universalis, Basel, pp. 149-167. Birkhäuser Verlag.

Avron, A. (2017). Self-extensional three-valued paraconsistent logics. Logica Universalis 11, $297-315$.

Avron, A. and J. Y. Béziau (2017). Self-extensional three-valued paraconsistent logics have no implication. Logic Journal of the IGPL 25(2), 183-194.

Avron, A. and A. Zamansky (2016). A paraconsistent view on B and S5. In L. Beklemishev, S. Demri, and A. Máté (Eds.), Advances in Modal Logic, Volume 11, London, pp. 21-37. College Publications.

Bell, J. L. (2005). Set Theory: Boolean-Valued Models and Independence Proofs (Third ed.), Volume 47 of Oxford Logic Guides. Oxford: Oxford University Press.

Benzmüller, C. (2019). Universal (meta-)logical reasoning: Recent successes. Science of Computer Programming 172, 48-62.

Béziau, J.-Y. (1998). Idempotent full paraconsistent negations are not algebraizable. Notre Dame Journal of Formal Logic 39(1), 135-139.

Béziau, J.-Y. (2002). S5 is a paraconsistent logic and so is first-order classical logic. Logical Studies 9, 301-309.

Béziau, J.-Y. (2005). Paraconsistent logic from a modal viewpoint. Journal of Applied Logic 3, $7-14$.

Blanchette, J. C. and T. Nipkow (2010). Nitpick: A counterexample generator for higher-order logic based on a relational model finder. In M. Kaufmann and L. C. Paulson (Eds.), ITP 2010, Volume 6172 of $L N C S$, pp. 131-146. Springer.

Blok, W. J. and D. Pigozzi (1989). Algebraizable Logics, Volume 77(396) of Memoirs of the American Mathematical Society. Providence, RI, USA: American Mathematical Society.

Bou, F., F. Esteva, J. M. Font, A. Gil, L. Godo, T. A., and V. Verdú (2009). Logics preserving degrees of truth from varieties of residuated lattices. Journal of Logic and Computation 19(6), $1031-1069$.

Bueno-Soler, J. and W. Carnielli (2017). Experimenting with consistency. In V. Markin and D. Zaitsev (Eds.), The Logical Legacy of Nikolai Vasiliev and Modern Logic, Volume 387 of Synthese Library (Studies in Epistemology, Logic, Methodology, and Philosophy of Science), Cham, pp. 199-221. Springer.

Carnielli, W. A. and M. E. Coniglio (2016a). Paraconsistent Logic: Consistency, Contradiction and Negation, Volume 40 of Logic, Epistemology, and the Unity of Science. Cham: Springer.

Carnielli, W. A. and M. E. Coniglio (2016b). Paraconsistent set theory by predicating on consistency. Journal of Logic and Computation 26(1), 97-116. First published online: 09 July 2013. 
Carnielli, W. A. and M. E. Coniglio (2020). Combining logics. In E. N. Zalta (Ed.), The Stanford Encyclopedia of Philosophy (Fall 2020 ed.). URL: http://plato.stanford.edu/archives/spr2014/entries/logic-combining/.

Carnielli, W. A. and M. E. Coniglio (2021). Twist-valued models for three-valued paraconsistent set theory. Logic and Logical Philosophy 30(2), 187-226.

Carnielli, W. A., M. E. Coniglio, D. M. Gabbay, P. Gouveia, and C. Sernadas (2008). Analysis and Synthesis of Logics: How to Cut and Paste Reasoning Systems, Volume 35 of Applied Logic Series. Springer.

Carnielli, W. A., M. E. Coniglio, and J. Marcos (2007). Logics of Formal Inconsistency. In D. M. Gabbay and F. Guenthner (Eds.), Handbook of Philosophical Logic (2nd. edition), Volume 14, pp. 1-93. Springer.

Carnielli, W. A., M. E. Coniglio, R. Podiacki, and T. Rodrigues (2014). On the way to a wider model theory: Completeness theorems for first-order logics of formal inconsistency. The Review of Symbolic Logic 7(3), 548-578.

Carnielli, W. A. and L. P. de Alcantara (1984). Paraconsistent algebras. Studia Logica 43(1/2), $79-88$.

Carnielli, W. A. and M. Lima-Marques (1999). Society semantics and multiple-valued logics. In W. A. Carnielli and I. M. D'Ottaviano (Eds.), Advances in Contemporary Logic and Computer Science. Proceedings of the XI Brazilian Conference on Mathematical Logic, May 1996, Salvador, Bahia, Brazil, Volume 235 of Contemporary Mathematics, pp. 33-52. Providence, RI, USA: American Mathematical Society.

Carnielli, W. A. and J. Marcos (2002). A taxonomy of C-systems. In W. A. Carnielli, M. E. Coniglio, and I. M. L. D'Ottaviano (Eds.), Paraconsistency: The Logical Way to the Inconsistent. Proceedings of the 2nd World Congress on Paraconsistency (WCP 2000), Volume 228 of Lecture Notes in Pure and Applied Mathematics, pp. 1-94. New York: Marcel Dekker.

Chellas, B. (1980). Modal Logic: An Introduction. New York: Cambridge University Press.

Coniglio, M., A. Figallo-Orellano, and A. C. Golzio (2020). First-order swap structures semantics for some logics of formal inconsistency. Journal of Logic and Computation 30(6), $1257-1290$.

Coniglio, M. and L. Prieto-Sanabria (2017). Modal logic S4 as a paraconsistent logic with a topological semantics. In C. C. P. Gouveia and F. Donisio (Eds.), Logic and Computation: Essays in Honour of Amilcar Sernadas, Volume 33 of Tributes, pp. 171-196. London: College Publications.

Coniglio, M. E., F. Esteva, and L. Godo (2014). Logics of formal inconsistency arising from systems of fuzzy logic. Logic Journal of the IGPL 22(6), 880-904.

Coniglio, M. E. and G. V. Toledo (2020). A simple decision procedure for da Costa's $C_{n}$ logics by Restricted Nmatrix semantics. arXiv:2011.10151 [math.LO].

Curry, H. B. (1963). Foundations of Mathematical Logic. New York: McGrawHill.

da Costa, N. C. A. (1963). Sistemas formais inconsistentes (Inconsistent formal systems, in Portuguese). Habilitation thesis, Universidade Federal do Paraná, Curitiba, Brazil. Republished by Editora UFPR, Curitiba, Brazil, 1993. 
da Costa, N. C. A. and M. Guillaume (1964). Sur les calculs Cn. Anais da Academia Brasileira de Ciências 36, 379-382.

de Araújo, A. L., E. H. Alves, and J. A. D. Guerzoni (1987). Some relations between modal and paraconsistent logic. The Journal of Non-Classical Logic 4(2), 33-44. Available at http://www.cle.unicamp.br/jancl//.

Došen, K. (1984). Negative modal operators in intuitionistic logic. Publications de l'Institut Mathématique. Nouvelle Série 35(49), 3-14.

Došen, K. (1986). Negation as a modal operator. Reports on Mathematical Logic 20, 15-27.

Dunn, J. M. (1993). Star and perp: Two treatments of negation. Philosophical perspectives 7, $331-357$.

Dunn, J. M. and G. M. Hardegree (2001). Algebraic Methods in Philosophical Logic, Volume 41 of Oxford Logic Guides. Oxford: Oxford University Press.

Dunn, J. M. and C. Zhou (2005). Negation in the context of gaggle theory. Studia Logica 80(23), $235-264$.

Ertola, R., F. Esteva, T. Flaminio, L. Godo, , and C. Noguera (2015). Exploring paraconsistency in degree-preserving fuzzy logics. Soft Computing 19(3), 531-546.

Fuenmayor, D. (2020). Topological semantics for paraconsistent and paracomplete logics. Archive of Formal Proofs. Available at https://isa-afp.org/entries/Topological_ Semantics.html,

Gabbay, D. M. (1975). A normal logic that is complete for neighborhood frames but not for Kripke frames. Theoria 41(3), 148-153.

Givant, S. and P. Halmos (2009). Introduction to Boolean Algebras. New York: Springer.

Henkin, L. (1949). Fragments of the propositional calculus. The Journal of Symbolic Logic 14(1), 42-48.

Jónsson, B. (1993). A survey of Boolean algebras with operators. In I. Rosenberg and G. Sabidussi (Eds.), Algebras and Orders, Volume 389 of NATO ASI Series (Series C: Mathematical and Physical Sciences), pp. 239-286. Dordrecht: Springer.

Lewin, R. A., I. F. Mikenberg, and M. G. Schwarze (1991). $C_{1}$ is not algebraizable. Notre Dame Journal of Formal Logic 32(4), 609-611.

Lewis, D. K. (1973). Counterfactuals. Cambridge, MA: Harvard University Press.

Eukasiewicz, J. (1948-1950). The shortest axiom of the implicational calculus of propositions. Proceedings of the Royal Irish Academy. Section A: Mathematical and Physical Sciences 52, 25-33.

MacNeille, H. (1937). Partially ordered sets. Transactions of the American Mathematical Society 42, 416-460.

Marcos, J. (2005a). Modality and paraconsistency. In M. Bilkova and L. Behounek (Eds.), The Logica Yearbook 2004, Proceedings of the XVIII International Symposium promoted by the Institute of Philosophy of the Academy of Sciences of the Czech Republic, pp. 213-222. Prague: Filosofia. 
Marcos, J. (2005b). Nearly every normal modal logic is paranormal. Logique et Analyse 48(189192), 279-300.

Montague, R. (1970). Universal grammar. Theoria 36, 373-398.

Mortensen, C. (1980). Every quotient algebra for $C_{1}$ is trivial. Notre Dame Journal of Formal Logic $21(4)$, 694-700.

Mortensen, C. (1989). Paraconsistency and $C_{1}$. In G. Priest, R. Routley, and J. Norman (Eds.), Paraconsistent Logic: Essays on the Inconsistent, pp. 289-305. Munich: Philosophia Verlag.

Nipkow, T., L. C. Paulson, and M. Wenzel (2002). Isabelle/HOL: a proof assistant for higherorder logic, Volume 2283 of Springer Science $\&$ Business Media. Berlin, Heidelberg: Springer.

Pacuit, E. (2017). Neighborhood semantics for modal logic. Cham: Springer.

Scott, D. (1970). Advice in modal logic. In K. Lambert (Ed.), Philosophical Problems in Logic: Some Recent Developments, Volume 29 of Synthese Library (Monographs on Epistemology, Logic, Methodology, Philosophy of Science, Sociology of Science and of Knowledge, and on the Mathematical Methods of Social and Behavioral Sciences), pp. 143-173. Dordrecht: D. Reidel.

Segerberg, K. (1982). Classical Propositional Operators: An exercise in the foundations of logic, Volume 5 of Oxford Logic Guides. Oxford: Clarendon Press.

Seoane, J. and L. P. de Alcantara (1991). On da Costa algebras. The Journal of Non-Classical Logic 8(2), 41-66. Available at http://www.cle.unicamp.br/jancl/.

Stone, M. H. (1936). The Theory of Representations of Boolean Algebras. Transactions of the American Mathematical Society 40, 37-111.

Sylvan, R. (1990). Variations on da Costa C Systems and Dual-Intuitionistic Logics I. Analyses of $C_{\omega}$ and $C C_{\omega}$. Studia Logica $49(1), 47-65$.

Tarski, A. (1937). Über additive und multiplikative Mengenkörper und Mengenfunktionen. Sprawozdania z Posiedzeń Towarzystwa Naukowego Warszawskiego Wydziat III Nauk Matematyczno-fizycznych (=Comptes Rendus des Séances de la Société des Sciences et des Lettres de Varsovie, Classe III ) 30, 151-181.

Urbas, I. (1987). On Brazilian Paraconsistent Logics. Ph. D. thesis, Australian National University, Canberra, AU.

Urbas, I. (1989). Paraconsistency and the C-Systems of da Costa. Notre Dame Journal of Formal Logic 30(4), 583-597.

Vakarelov, D. (1989). Consistency, completeness and negation. In G. Priest, R. Routley, and J. Norman (Eds.), Paraconsistent Logic: Essays on the Inconsistent, pp. 328-363. Munich: Philosophia Verlag.

Zanardo, A., A. Sernadas, and C. Sernadas (2001). Fibring: Completeness preservation. The Journal of Symbolic Logic 66(1), 414-439. 